\title{
Light-front Ward-Takahashi identity for two-fermion systems
}

\author{
J. A. O. Marinho, ${ }^{1}$ T. Frederico, ${ }^{1}$ E. Pace, ${ }^{2}$ G. Salmè ${ }^{3}$ and P. U. Sauer ${ }^{4}$ \\ ${ }^{1}$ Departamento de Física, Instituto Tecnológico de Aeronáutica, 12.228-900 São José dos Campos, São Paulo, Brazil \\ ${ }^{2}$ Dipartimento di Fisica, Università di Roma "Tor Vergata" and Istituto Nazionale di Fisica Nucleare, Sezione Tor Vergata, \\ Via della Ricerca Scientifica 1, I-00133 Roma, Italy \\ ${ }^{3}$ Istituto Nazionale di Fisica Nucleare, Sezione Roma I, Piazzale A. Moro 2, I-00185 Roma, Italy \\ ${ }^{4}$ Institute for Theoretical Physics, Leibniz University, D-30167 Hannover, Germany
}

(Received 3 April 2008; published 23 June 2008)

\begin{abstract}
We propose a three-dimensional electromagnetic current operator within light-front dynamics that satisfies a light-front Ward-Takahashi identity for two-fermion systems. The light-front current operator is obtained by a quasipotential reduction of the four-dimensional current operator and acts on the light-front valence component of bound or scattering states. A relation between the light-front valence wave function and the four-dimensional Bethe-Salpeter amplitude both for bound or scattering states is also derived, such that the matrix elements of the four-dimensional current operator can be fully recovered from the corresponding light-front ones. The light-front current operator can be perturbatively calculated through a quasipotential expansion, and the divergence of the proposed current satisfies a Ward-Takahashi identity at any given order of the expansion. In the quasipotential expansion the instantaneous terms of the fermion propagator are accounted for by the effective interaction and two-body currents. We exemplify our theoretical construction in the Yukawa model in the ladder approximation, investigating in detail the current operator at the lowest nontrivial order of the quasipotential expansion of the Bethe-Salpeter equation. The explicit realization of the light-front form of the Ward-Takahashi identity is verified. We also show the relevance of instantaneous terms and of the pair contribution to the two-body current and the Ward-Takahashi identity.
\end{abstract}

DOI: 10.1103/PhysRevD.77.116010

PACS numbers: 11.10.St, 11.40.- q, 13.40.-f, 21.45.-v

\section{INTRODUCTION}

A detailed investigation of the electromagnetic (em) properties of hadrons (including also nuclei) requests a careful treatment of the current operator for interactingfermion systems. In particular the gauge symmetry plays the well-known essential role, that formally leads to the fulfillment of the Ward-Takahashi identity (WTI). Following the seminal paper by Gross and Riska [1], in a fully covariant theory, namely, in a field-theoretical framework in its full glory, one should first solve the BetheSalpeter (BS) equation and obtain a consistent current operator; then through a Mandelstam [2] approach, one should evaluate the hadron em properties. Unfortunately the BS equation can be solved only within approximate schemes or for simple examples (see, e.g., [3-7]). For instance, an appealing technique for solving the BS equation is based on the quasipotential (QP) expansion of the four-dimensional $T$ matrix (see, e.g., [8]), where one introduces an auxiliary four-dimensional free Green's function (in [8] it was three-dimensional), hopefully clever enough to allow a meaningful truncation of the expansion. Then, one projects the relevant operators onto a threedimensional hyperplane for obtaining a three-dimensional integral equation for both scattering and bound states. These three-dimensional states allow to fully reconstruct the four-dimensional BS amplitude solution after applying proper reverse projection operators $[9,10]$. The method is in principle exact, giving the solution of the fourdimensional BS equation if the QP is evaluated without any approximation. As shown in [11] for massive particles, with a proper choice of the auxiliary free Green's function the QP expansion can be put in correspondence with the decomposition of the wave function in terms of Fock components on the light-front (LF) hyperplane, i. e. on the three-dimensional space adopted in this paper. There are extended reviews [12-14] illustrating the relevant features of the LF framework, introduced in a famous paper by Dirac [15], and the interested reader can profit of those.

The present paper is a part of a program which attempts: (i) to solve the four-dimensional $\mathrm{BS}$ equation for two interacting particles, both bosons and fermions, by using the QP expansion and a LF three-dimensional projection onto the LF hypersurface (see, $[9,10,16]$ for previous works) and, (ii) to develop a procedure for evaluating matrix elements of the four-dimensional em current keeping WTI valid, at any order in the truncation. A key role is played by the LF reverse projection operator that allows the full reconstruction of the four-dimensional BS amplitudes, without loosing any physics content $[9,10,16]$. The price to be paid, however, is a rather complicated effective threedimensional interaction, that is derived from the full fourdimensional one. In particular, the effective threedimensional interaction is obtained from the proper projection of the quasipotential, expanded in powers of the four-dimensional interaction present in the BS equation. In 
conclusion, such an approach is useful if the convergence rate of the expansion allows to adopt a truncated series, at a nontrivial low order, in the actual calculations. The procedure of LF projection and expansion/truncation of the three-dimensional interaction is discussed in Ref. [9] for the two-boson case and in Ref. [10] for the two-fermion case.

By using the two previously introduced ingredients, namely, the QP approach and LF projection, we first investigate the possibility to express the matrix elements of the four-dimensional em current operator, for an interacting two-fermion system, through matrix elements of a three-dimensional LF current operator between valence states, without introducing any truncation (see, [17] for a formal definition of the valence state). In particular, it will be shown that the three-dimensional LF current operator fulfills WTI. This initial analysis will allow us to define LF charge operators and the form of the WTI onto the LF hyperplane. After introducing a proper truncation procedure of the LF current operator, it will be presented a systematical way for constructing workable approximations of the matrix elements of the LF current by increasing the order of truncation of the QP expansion. It will be shown that the truncated LF current fulfills WTI with the LF charge operators obtained in the nontruncated case, leaving a complete study of the relativistic covariance under dynamical transformations to a future investigation. In order to illustrate our procedure in an actual case, it will be analyzed the lowest nontrivial truncation of a Yukawa model with chargeless boson exchange, in ladder approximation.

As is well-known (see, e.g., Ref. [14]), a peculiar feature of any LF description of fermionic systems is the so-called instantaneous (in LF time) propagation. The treatment of this issue makes sharply different the study of the em current for fermionic systems from the bosonic case, already analyzed in detail in Ref. [16], and in what follows this point will be often emphasized.

In general, the construction of a conserved current operator acting on the valence component of the LF wave function is a challenging problem. For two-boson systems Kvinikhidze and Blankleider [18] developed gauging techniques for solving this problem. They were able to obtain a current that satisfies a WTI and therefore is conserved. It is remarkable that for the two-boson case the current operator derived in Ref. [16] is equivalent to the one obtained with gauging techniques [18]. An earlier perturbative approach to the WTI within LF quantization was performed in Ref. [19].

This work is organized as follows. In Sec. II, the fourdimensional expression of the em current operator and the associated WTI in the context of BS formalism, that leads to current conservation, is briefly recalled. In Sec. III, we illustrate the LF-time projection technique, that allows one to eliminate relative LF-time, within a QP approach ap- plied to the BS equation for two-fermion systems. In Sec. IV, it is shown the connection of the two-fermion valence LF wave function, for bound and scattering states, with the corresponding BS amplitude. In Sec. V, the threedimensional current operator is introduced and the fulfilled WTI is discussed. In Sec. VI, we propose a truncated form of the em current operator that satisfies the WTI at any given order, and we explicitly show that the current conservation is fulfilled by the matrix elements calculated between valence states. In Sec. VII, we present the formal construction in an actual case: a Yukawa model with a chargeless boson exchange, in ladder approximation. Our conclusions are drawn in Sec. VIII.

\section{FOUR-DIMENSIONAL EM CURRENT OPERATOR}

The general field-theoretical description of an interacting two-fermion system is given by the BS equation, where the driving term, the interaction $V(K)$, is assumed to be based on the irreducible exchange of bosons. In what follows self-energy corrections will be omitted and considered elsewhere. Let us remind the reader that the total four-momentum $K$ is conserved, and all BS operators, as $V(K)$, depend parametrically on $K$ (see, e.g., [16]). As is well-known, the interaction $V(K)$ yields the transition matrix $T(K)$, i.e.,

$$
T(K)=V(K)+V G_{0}(K) T(K),
$$

and the full Green's function, $G(K)$, i.e.,

$$
\begin{aligned}
G(K) & =G_{0}(K)+G_{0}(K) V(K) G(K) \\
& =G_{0}(K)+G_{0}(K) T(K) G_{0}(K),
\end{aligned}
$$

where $G_{0}(K)$ is the free Green's function of two fermions, with four-momentum operators $\hat{k}_{j}(j=1,2)$, viz.,

$$
G_{0}(K)=\frac{l^{2}}{2 \pi} \frac{\hat{k}_{1}+m_{1}}{\hat{k}_{1}^{2}-m_{1}^{2}+i \varepsilon} \frac{\hat{k}_{2}+m_{2}}{\hat{k}_{2}^{2}-m_{2}^{2}+i \varepsilon},
$$

and $\hat{k}_{2}=K-\hat{k}_{1}$. For convenience a factor of $1 / 2 \pi$ has been included in the above definition.

The BS amplitude $|\Psi\rangle$, dependent upon internal variables, satisfies (the $\lim _{\varepsilon \rightarrow 0}$ is understood)

$$
G^{-1}(K)|\Psi\rangle=0,
$$

with appropriate boundary conditions for bound and scattering states [7].

The em current operator, $\mathcal{J}^{\mu}$, corresponding to the interaction $V(K)$ has a free term, $\mathcal{J}_{0}^{\mu}$, and another one, $\mathcal{J}_{I}^{\mu}$, which depends on the interaction, as dictated by the commutation rules between $\mathcal{J}^{\mu}$ and the generators of the Poincarè group, i.e.,

$$
\mathcal{J}^{\mu}(Q)=\mathcal{J}_{0}^{\mu}(Q)+\mathcal{J}_{I}^{\mu}(Q),
$$

where $\mathcal{J}_{I}^{\mu}$ depends parametrically on the four-momentum 
transfer $Q=K_{f}-K_{i}$. In particular the Lorentz covariance of $\mathcal{J}^{\mu}$ imposes that (see, e.g., [20])

$$
\Lambda_{\nu}^{\mu} \mathcal{J}^{\nu}\left(Q^{\prime}\right)=D^{J_{f}}\left[W\left(\Lambda^{-1}, K_{f}\right)\right]^{-1} \mathcal{J}^{\mu}(Q) D^{J_{i}}\left[W\left(\Lambda^{-1}, K_{i}\right)\right]
$$

where $\Lambda$ is a Lorentz transformation, $Q^{\prime}=\Lambda^{-1} Q, J_{i(f)}$ is the total spin of the initial (final) system, and $D^{J}$ is the unitary representation of the Wigner rotation, $W\left(\Lambda^{-1}, K\right)$, corresponding to $\Lambda$. As to the BS amplitude one has

$$
\begin{aligned}
\Psi_{J M_{J}}^{\prime}\left(k_{1}^{\prime}, K^{\prime}\right)= & \sum_{M_{J}^{\prime}} S_{1}^{-1}(\Lambda) S_{2}^{-1}(\Lambda) D_{M_{J}^{\prime} M_{J}}^{J}\left[W\left(\Lambda^{-1}, K\right)\right] \\
& \times \Psi_{J M_{J}^{\prime}}\left(k_{1}, K\right)
\end{aligned}
$$

where $k_{1}^{\prime}=\Lambda^{-1} k_{1}, K^{\prime}=\Lambda^{-1} K$, and $S_{1(2)}(\Lambda)$ is the spinorial representation of the transformation $\Lambda$ [21].

Notably, the four current satisfies the following WardTakahashi identity (see, e.g., $[1,7]$ )

$$
Q_{\mu} \mathcal{J}^{\mu}(Q)=G^{-1}\left(K_{f}\right) \hat{e}-\hat{e} G^{-1}\left(K_{i}\right),
$$

where $\hat{e}=\hat{e}_{1}+\hat{e}_{2}$ and $\hat{e}_{j}$ is the charge operator for the fermion $j$, with matrix elements given by

$$
\left\langle k_{j}\left|\hat{e}_{j}\right| p_{j}\right\rangle=e_{j} \delta^{4}\left(k_{j}-p_{j}-Q\right) \text {. }
$$

Since the inverse of the full interacting Green's function is given by

$$
G^{-1}(K)=G_{0}^{-1}(K)-V(K)
$$

and

$$
Q_{\mu} \mathcal{J}_{0}^{\mu}(Q)=G_{0}^{-1}\left(K_{f}\right) \hat{e}-\hat{e} G_{0}^{-1}\left(K_{i}\right)
$$

then the interacting current fulfills the following relation

$$
Q_{\mu} \mathcal{J}_{I}^{\mu}(Q)=\hat{e} V\left(K_{i}\right)-V\left(K_{f}\right) \hat{e} .
$$

Once we consider the matrix elements $\left\langle\Psi_{f}\left|\mathcal{J}^{\mu}(Q)\right| \Psi_{i}\right\rangle$, current conservation can be explicitly obtained through Eq. (4), i.e.,

$$
\begin{aligned}
Q_{\mu}\left\langle\Psi_{f}\left|\mathcal{J}^{\mu}(Q)\right| \Psi_{i}\right\rangle & =\left\langle\Psi_{f}\left|\left[G^{-1}\left(K_{f}\right) \hat{e}-\hat{e} G^{-1}\left(K_{i}\right)\right]\right| \Psi_{i}\right\rangle \\
& =0 .
\end{aligned}
$$

\section{LF-TIME PROJECTION AND THE QUASIPOTENTIAL APPROACH FOR FERMIONS}

Following Ref. [10], where the LF projection of the BS equation for a fermionic system was investigated, here we briefly resume the QP formalism for the LF projection of the two-fermion BS equation (see also, Refs. [22-24]), and we introduce a more compact and efficient operator notation.

Crucial for the LF projection is the separation of the fermion propagator in an on-shell term and in an instantaneous one, viz.,

$$
\frac{k+m}{k^{2}-m^{2}+i \varepsilon}=\frac{k_{\mathrm{on}}+m}{k^{+}\left(k^{-}-k_{\mathrm{on}}^{-}+\frac{i \varepsilon}{k^{+}}\right)}+\frac{\gamma^{+}}{2 k^{+}},
$$

where $k_{\mathrm{on}}^{-}=\left(\vec{k}_{\perp}^{2}+m^{2}\right) / k^{+}$is the on-minus-shell momentum. The second term in Eq. (14) does not lead to a freepropagation in the global time, since the Fourier transform is divergent and yields $\delta\left(x^{+}\right)$, i.e., an instantaneous (in the LF time) propagation. This term makes the treatment of a fermionic system basically different from the treatment of a bosonic one. The LF projection is based on the on-shell part of the two-fermion free propagator of Eq. (3), i.e.,

$$
\bar{G}_{0}(K):=\frac{i^{2}}{2 \pi} \frac{\left(\hat{k}_{1 \mathrm{on}}+m_{1}\right)\left(\hat{k}_{2 \mathrm{on}}+m_{2}\right)}{\hat{k}_{1}^{+}\left(K^{+}-\hat{k}_{1}^{+}\right)\left(\hat{k}_{1}^{-}-\frac{\hat{\vec{k}}_{1 \perp}^{2}+m_{1}^{2}-i \varepsilon}{\hat{k}_{1}^{+}}\right)\left(K^{-}-\hat{k}_{1}^{-}-\frac{\hat{\hat{k}}_{2 \perp}^{2}+m_{2}^{2}-i \varepsilon}{K^{+}-\hat{k}_{1}^{+}}\right)} .
$$

The role of $\bar{G}_{0}(K)$ will become more and more clear as the LF integral equations for the transition matrix, the valence Green's function, the valence wave function, and etc., will be derived. It should be pointed out that the instantaneous terms are fully restored into the theory through the three-dimensional effective interaction (see, below and also Sec. IV). Once the full structure of the propagator is taken into account, the matrix elements of the three-dimensional em current operator become equal to the corresponding four-dimensional quantities, as will be discussed in Secs. IV and V.

The free Green's functions $G_{0}(K)$ and $\bar{G}_{0}(K)$ are fourdimensional operators which depend upon the fourmomenta of the two fermions. Let us introduce the LF free Green's function, $g_{0}(K)$, that is a three-dimensional operator which depends upon the LF momenta $\left(k_{i}^{+}, \vec{k}_{i \perp}\right)$ only. It is obtained from the four-dimensional on-shell Green's function by projection, i.e.,

$$
\begin{aligned}
g_{0}(K)=\left|\bar{G}_{0}(K)\right|:=\int d k_{1}^{\prime-} d k_{1}^{-}\left\langle k_{1}^{\prime-}\left|\bar{G}_{0}(K)\right| k_{1}^{-}\right\rangle \\
=i \theta\left(K^{+}-\hat{k}_{1}^{+}\right) \theta\left(\hat{k}_{1}^{+}\right) \\
\quad \times \frac{2 m_{1} 2 m_{2} \Lambda_{+}\left(\hat{k}_{1 \text { on }}\right) \Lambda_{+}\left(\hat{k}_{2 \text { on }}\right)}{\hat{k}_{1}^{+}\left(K^{+}-\hat{k}_{1}^{+}\right)\left(K^{-}-\hat{k}_{\text {lon }}^{-}-\hat{k}_{2 \text { on }}^{-}+i \varepsilon\right)},
\end{aligned}
$$

where one can choose $K^{+}>0$ without any loss of generality, and $\Lambda_{+}\left(\hat{k}_{\text {on }}\right)=\left(\hat{k}_{\text {on }}+m\right) / 2 m$ is the positive energy spinor projector. The vertical bar, |, on the right (left) indicates that the minus component present in the ket, $\left|k^{-}\right\rangle$(in the bra, $\left\langle k^{-}\right|$) is integrated out, namely, through the vertical bar operation one projects on the LF hypersur- 
face $x^{+}=0$ (see, Refs. $[9,10,16]$ ). Thus, in this paper the two vertical bars in Eq. (16) do not mean the absolute value; they indicate, indeed, the transition from a fourdimensional operator to a three-dimensional one. It is worth noting that $g_{0}(K)$ yields the free global propagation of two on-shell fermions, and its inverse exists in the valence sector, since $\Lambda_{+}\left(\hat{k}_{1 \text { on }}\right) \Lambda_{+}\left(\hat{k}_{2 \text { on }}\right)$ is the representation of the identity in a two-particle spinor space. Furthermore, one can express the three-dimensional $g_{0}^{-1}(K)$ in terms of the corresponding four-dimensional quantity, $G_{0}^{-1}(K)$, as follows [see, Appendix A, Eq. (A6)]

$$
g_{0}^{-1}(K)=\bar{\Pi}_{0}(K) G_{0}^{-1}(K) \Pi_{0}(K)
$$

where we define the free reverse $L F$ projection operator [cf. Eq. (23)] and its LF conjugated, as

$$
\Pi_{0}(K):=\bar{G}_{0}(K)\left|g_{0}^{-1}(K) \quad \bar{\Pi}_{0}(K):=g_{0}^{-1}(K)\right| \bar{G}_{0}(K) .
$$

An explicit expression of $\Pi_{0}(K)$ can be found in the Appendix of Ref. [10]. These operators have the following properties, as shown by the definitions in Eq. (19) and from Eq. (A6),

$$
\begin{array}{cl}
\mid \Pi_{0}(K)=\mathrm{I} & \bar{\Pi}_{0}(K) \mid=\mathrm{I} \\
\mid \bar{G}_{0}(K) G_{0}^{-1}(K) \Pi_{0}(K)=\mathrm{I} & \bar{\Pi}_{0}(K) G_{0}^{-1}(K) \bar{G}_{0}(K) \mid=\mathrm{I}
\end{array}
$$

where I is the identity in the LF three-dimensional space. The LF free state, $\left|\phi_{0}\right\rangle$, is a solution of

$$
g_{0}^{-1}(K)\left|\phi_{0}\right\rangle=0
$$

then from Eq. (18) one has

$$
\bar{\Pi}_{0}(K) G_{0}^{-1}(K) \Pi_{0}(K)\left|\phi_{0}\right\rangle=0 .
$$

The last equation leads to the following transformation property between the noninteracting BS amplitude and the corresponding LF three-dimensional state, viz.,

$$
\left|\Psi_{0}\right\rangle=\Pi_{0}(K)\left|\phi_{0}\right\rangle
$$

Indeed, one obtains the eigenequation for $\left|\Psi_{0}\right\rangle$ by applying $G_{0}^{-1}(K)$ to Eq. (23) and performing the understood $\lim _{\varepsilon \rightarrow 0}$. Then, on the right-hand side (rhs) of Eq. (23) one can use Eqs. (19) and (A3) (that holds for any $\varepsilon$ ) and can shift $\lim _{\varepsilon \rightarrow 0}$ to $g_{0}^{-1}(K)\left|\phi_{0}\right\rangle$, namely, one has

$$
G_{0}^{-1}(K)\left|\Psi_{0}\right\rangle=0 .
$$

Furthermore, one gets two expressions of the inverse of Eq. (23) by using Eqs. (20) and (23), viz.,

$$
\left.\| \Psi_{0}\right\rangle=\left|\phi_{0}\right\rangle
$$

and

$$
\left.\left|\bar{G}_{0}(K) G_{0}^{-1}(K)\right| \Psi_{0}\right\rangle=\left|\phi_{0}\right\rangle .
$$

In Eq. (26) the understood $\lim _{\varepsilon \rightarrow 0}$ affects the whole left- hand side. Notably, from the uniqueness of the solutions of Eq. (24) and applying Appendix A of Ref. [10], one can demonstrate that for any given solution $\left|\Psi_{0}\right\rangle$ of Eq. (24) one has a LF wave function obtained by Eq. (26), that in turn fulfills the eigenequation (21) and yields the initial $\left|\Psi_{0}\right\rangle$ through Eq. (23). Namely, there is a one-to-one relation between a given four-dimensional BS amplitude $\left|\Psi_{0}\right\rangle$ and the corresponding three-dimensional $\left|\phi_{0}\right\rangle$. The noninteracting operators $\Pi_{0}(K)$ and $\bar{\Pi}_{0}(K)$ connect threeand four-dimensional quantities. In the next section the corresponding interacting operators will be given.

The QP formalism makes use of the four-dimensional auxiliary Green's function $\tilde{G}_{0}(K)$ defined by

$$
\begin{aligned}
\tilde{G}_{0}(K) & :=\bar{G}_{0}(K)\left|g_{0}^{-1}(K)\right| \bar{G}_{0}(K)=\Pi_{0}(K) g_{0}(K) \bar{\Pi}_{0}(K) \\
& =\bar{G}_{0}(K)\left|\bar{\Pi}_{0}(K)=\Pi_{0}(K)\right| \bar{G}_{0}(K) .
\end{aligned}
$$

This operator has the following useful properties

$$
\left|\tilde{G}_{0}(K)=\right| \bar{G}_{0}(K) \quad \tilde{G}_{0}(K)\left|=\bar{G}_{0}(K)\right| .
$$

Let us apply the QP formalism to the four-dimensional transition matrix (see Refs. [8-10]), i.e.,

$$
\begin{aligned}
T(K) & =W(K)+W(K) \tilde{G}_{0}(K) T(K) \\
& =W(K)+T(K) \tilde{G}_{0}(K) W(K),
\end{aligned}
$$

where the effective interaction $W(K)$, in turn, is a solution of

$$
W(K)=V(K)+V(K) \Delta_{0}(K) W(K),
$$

with

$$
\Delta_{0}(K):=G_{0}(K)-\tilde{G}_{0}(K) .
$$

The four-dimensional quantity $\Delta_{0}(K)$ clearly contains the instantaneous terms and has the following properties

$$
\gamma_{1}^{+} \gamma_{2}^{+} \Delta_{0}(K)\left|=0 \quad \gamma_{1}^{+} \gamma_{2}^{+}\right| \Delta_{0}(K)=0 .
$$

It is worth noting that in Eq. (29) the driving term $V(K)$ is substituted by $W(K)$, in order to increase the rate of convergence of the iterative solution of the transition matrix (for numerical studies of the convergence see, e.g., $[9,25]$ ). In a similar manner, Eq. (30) can be solved by iteration, obtaining

$$
W(K)=\sum_{i=1}^{\infty} W_{i}(K)
$$

with $W_{i}(K)=V(K)\left[\Delta_{0}(K) V(K)\right]^{i-1}$. The physical meaning of the convergence of this series will be discussed in Sec. VI.

The three-dimensional LF transition matrix can be introduced through (see also, [10])

$$
t(K)=\bar{\Pi}_{0}(K) T(K) \Pi_{0}(K) .
$$

Then, by using Eq. (29), one has 


$$
\begin{aligned}
t(K) & =\bar{\Pi}_{0}(K)\left[W(K)+W(K) \tilde{G}_{0}(K) T(K)\right] \Pi_{0}(K) \\
& ==w(K)+w(K) g_{0}(K) t(K) \\
& =w(K)+w(K) g(K) w(K),
\end{aligned}
$$

where the three-dimensional driving term, $w(K)$ is obtained from the four-dimensional interaction $W(K)$ according to

$$
w(K):=\bar{\Pi}_{0}(K) W(K) \Pi_{0}(K),
$$

and the three-dimensional interacting Green's function is given by

$$
g(K)=g_{0}(K)+g_{0}(K) t(K) g_{0}(K) .
$$

Note that (i) the positions of $\bar{\Pi}_{0}(K)$ and $\Pi_{0}(K)$ in Eq. (34), leading to the integrations over the external minus components, straightforwardly indicate that $t(k)$ is a threedimensional quantity [for comparison see Eq. (27), where the four-dimensional quantity $\tilde{G}_{0}(K)$ is defined, and the integrations are on the internal minus components]; (ii) the effective interaction $w(K)$ contains the coupling of the valence sector to the higher Fock-state components of the wave function through $W(K)$ [11].

Finally, the four-dimensional $T(K)$ can be rewritten as follows

$$
T(K)=W(K)+W(K) \Pi_{0}(K) g(K) \bar{\Pi}_{0}(K) W(K) .
$$

Such an expression is useful to discuss the relation between the four-dimensional BS amplitude for a bound state and the corresponding three-dimensional valence wave function (cf. Sec. IV).

The LF interacting Green's function, or resolvent, can also be written as a function of $w(K)$ as follows:

$$
\begin{aligned}
g(K) & =g_{0}(K)+g_{0}(K) w(K) g(K) \\
& =g_{0}(K)+g(K) w(K) g_{0}(K) .
\end{aligned}
$$

It should be pointed out that $g(K)$ is the Fourier transform of the global propagator of two interacting fermions between two LF hypersurfaces with given $x^{+}$'s. Its inverse can be easily related to the four-dimensional $G^{-1}(K)$ by using Eqs. (18), (30), and (36). As a matter of fact, one has

$$
\begin{aligned}
g^{-1}(K)= & g_{0}^{-1}(K)-w(K) \\
= & \bar{\Pi}_{0}(K)\left[G_{0}^{-1}(K)-W(K)\right] \Pi_{0}(K) \\
= & \bar{\Pi}_{0}(K)\left[G_{0}^{-1}(K)-V(K)(1\right. \\
& \left.\left.+\Delta_{0}(K) W(K)\right)\right] \Pi_{0}(K) \\
= & \bar{\Pi}_{0}(K) G^{-1}(K)\left[1+\Delta_{0}(K) W(K)\right] \Pi_{0}(K) \\
& -\bar{\Pi}_{0}(K) G_{0}^{-1}(K) \Delta_{0}(K) W(K) \Pi_{0}(K) .
\end{aligned}
$$

The last term is vanishing, as can be shown by using Eqs. (19) and (A4) and the second relation in Eq. (32). Finally one obtains

$$
g^{-1}(K)=\bar{\Pi}_{0}(K) G^{-1}(K) \Pi(K)
$$

where

$$
\Pi(K)=\left[1+\Delta_{0}(K) W(K)\right] \Pi_{0}(K)
$$

is the interacting $L F$ reverse projection operator. From analogous steps one can obtain the corresponding LF conjugated operator, given by

$$
\bar{\Pi}(K)=\bar{\Pi}_{0}(K)\left[1+W(K) \Delta_{0}(K)\right] .
$$

It will be very useful in what follows to note that by using Eqs. (20), (32), (A3), and (A4) one has in the threedimensional space

$$
\left|\bar{G}_{0}(K) G_{0}^{-1}(K) \Pi(K)=\mathrm{I} \quad \bar{\Pi}(K) G_{0}^{-1}(K) \bar{G}_{0}(K)\right|=\mathrm{I} .
$$

The solution of the following three-dimensional equation,

$$
g^{-1}(K)|\phi\rangle=\left[g_{0}^{-1}(K)-w(K)\right]|\phi\rangle=0
$$

with appropriate boundary conditions for bound and scattering states, is the valence component of the LF wave function $[13,14]$. It turns out that the full complexity of the Fock space comes through the effective interaction. However, the truncation of the quasipotential [see, Eq. (33)] limits the number of Fock components involved in the construction of effective interaction to be used to obtain the valence wave function [11]. Then, one could argue that the convergence rate of the QP expansion is related to the smallness of the probability for the higher Fock-components.

Inserting Eq. (41) in the eigenequation (45) one has

$$
g^{-1}(K)|\phi\rangle=\bar{\Pi}_{0}(K) G^{-1}(K) \Pi(K)|\phi\rangle=0
$$

that leads to the following relation between the threedimensional valence component and the four-dimensional BS amplitude

$$
|\Psi\rangle=\Pi(K)|\phi\rangle .
$$

Note that $|\Psi\rangle$ fulfills Eq. (4) (as can be seen by using the results in Appendix A of [10]). Furthermore, by applying Eq. (44), one gets

$$
\left.\left|\bar{G}_{0}(K) G_{0}^{-1}(K)\right| \Psi\right\rangle=|\phi\rangle .
$$

In the next section more details will be given about the oneto-one relation between the four-dimensional $|\Psi\rangle$ and the three-dimensional $|\phi\rangle$.

Finally, let us remind the reader that, the on-mass-shell matrix elements of $T(K)$, which define the two-fermion scattering amplitude are identical to the ones obtained from $t(K)[10]$. 


\section{THE LF VALENCE WAVE FUNCTION AND THE BS AMPLITUDE}

In this section, we will analyze the interacting operator $\Pi(K)$, Eq. (42), that generates the full BS amplitude, for bound and scattering states of two-fermion systems, starting from the corresponding valence wave functions. It should be emphasized the key role played by $\Pi(K)$, when the matrix elements of a four-dimensional operator acting on the BS amplitudes are considered. In particular, the LF reverse projection allows to express those matrix elements in terms of matrix elements of effective operators acting on the valence wave functions.

The relation between the BS amplitude for a twofermion bound system, $\left|\Psi_{B}\right\rangle$, and the corresponding valence wave function has been derived in [10], and reads as

$$
\left|\Psi_{B}\right\rangle=G_{0}\left(K_{B}\right) W\left(K_{B}\right) \Pi_{0}\left(K_{B}\right)\left|\phi_{B}\right\rangle .
$$

This can be obtained through the analysis of the poles of Eq. (38).

Since the valence wave function is the solution of

$$
\left|\phi_{B}\right\rangle=g_{0}\left(K_{B}\right) w\left(K_{B}\right)\left|\phi_{B}\right\rangle,
$$

the vanishing quantity $\left.\bar{G}_{0}\left(K_{B}\right)\left|\left(g_{0}^{-1}\left(K_{B}\right)-w\left(K_{B}\right)\right)\right| \phi_{B}\right\rangle=$ 0 can be added to Eq. (49) in order to recover the LF reverse projection as given in Eq. (42), i.e.,

$$
\left|\Psi_{B}\right\rangle=\left[1+\Delta_{0}\left(K_{B}\right) W\left(K_{B}\right)\right] \Pi_{0}\left(K_{B}\right)\left|\phi_{B}\right\rangle=\Pi\left(K_{B}\right)\left|\phi_{B}\right\rangle .
$$

Therefore any $\left|\Psi_{B}\right\rangle$ can be generated through $\Pi\left(K_{B}\right)$ from the corresponding valence wave function $\left|\phi_{B}\right\rangle$, eigensolution of Eq. (45). Moreover, from Eq. (48) one has

$$
\left.\left|\bar{G}_{0}\left(K_{B}\right) G_{0}^{-1}\left(K_{B}\right)\right| \Psi_{B}\right\rangle=\left|\phi_{B}\right\rangle .
$$

Let us now show that the operator $\Pi(K)$ connects BS amplitudes and valence wave functions for scattering states, as well. The BS amplitude for scattering states satisfies the four-dimensional Lippman-Schwinger type inhomogeneous equation,

$$
\begin{aligned}
\left|\Psi^{+}\right\rangle & =\left|\Psi_{0}\right\rangle+G_{0}(K) T(K)\left|\Psi_{0}\right\rangle \\
& =\left[1+G_{0}(K) T(K)\right] \Pi_{0}\left|\phi_{0}\right\rangle,
\end{aligned}
$$

where we have made use of Eq. (23), that univocally relates $\left|\Psi_{0}\right\rangle$ to $\left|\phi_{0}\right\rangle$. In the three-dimensional space the scattering solution of Eq. (45) is given by

$$
\begin{aligned}
\left|\phi^{+}\right\rangle & =[1+g(K) w(K)]\left|\phi_{0}\right\rangle \\
& =\left[g_{0}(K)+g(K) w(K) g_{0}(K)\right] g_{0}^{-1}(K)\left|\phi_{0}\right\rangle \\
& =g(K) g_{0}^{-1}(K)\left|\phi_{0}\right\rangle,
\end{aligned}
$$

which implies the formal identity $g_{0}(K) g^{-1}(K)\left|\phi^{+}\right\rangle=$ $\left|\phi_{0}\right\rangle$. Then, by using Eq. (B1), one gets

$$
\begin{aligned}
\left|\Psi^{+}\right\rangle & =\left[1+G_{0}(K) T(K)\right] \Pi_{0}(K)\left|\phi_{0}\right\rangle \\
& =\left[1+G_{0}(K) T(K)\right] \Pi_{0}(K) g_{0}(K) g^{-1}(K)\left|\phi^{+}\right\rangle \\
& =\Pi(K)\left|\phi^{+}\right\rangle .
\end{aligned}
$$

From Eq. (48), the valence component of the LF wave function can be obtained directly from the BS amplitude, i.e.,

$$
\left.\left|\bar{G}_{0}(K) G_{0}^{-1}(K)\right| \Psi^{+}\right\rangle=\left|\phi^{+}\right\rangle .
$$

It is worth noting that the operator $\mid \bar{G}_{0}(K) G_{0}^{-1}(K)$ cuts the instantaneous terms and projects onto the LF hyperplane (through the $k^{-}$integration), while $\Pi(K)$ reconstructs the full structure of the four-dimensional BS amplitude through the instantaneous terms contained in $G_{0}$ (see, $\Delta_{0}$ ) and $W$. Moreover, the instantaneous terms affect the effective interaction $w$ that determines $\left|\phi_{B}\right\rangle$ and $\left|\phi^{+}\right\rangle$ [cf. Eq. (45)].

We should point out that the normalization of the BS amplitude for a bound state [26] expressed in an operatorial form, $\mathcal{N}(K)$, can be mapped onto an expectation value of a proper three-dimensional operator viz., $\langle\Psi|\mathcal{N}(K)| \Psi\rangle=$ $\langle\phi|\bar{\Pi}(K) \mathcal{N}(K) \Pi(K)| \phi\rangle$. Note that on one side, the BS amplitude normalization corresponds to the sum over the probabilities of each Fock component in the full LF wave function [17], and on the other side the full complexity of the Fock space is summarized in the three-dimensional operator $\bar{\Pi}(K) \mathcal{N}(K) \Pi(K)$.

\section{LF WARD-TAKAHASHI IDENTITY}

Once we have the relation between the four-dimensional BS amplitude and the three-dimensional LF valence wave function [see, Eq. (47)], the LF em current operator can be obtained from the matrix element of the four-dimensional current. As a matter of fact, one has for both scattering and bound states

$$
\left\langle\Psi_{f}\left|\mathcal{J}^{\mu}(Q)\right| \Psi_{i}\right\rangle=\left\langle\phi_{f}\left|j^{\mu}\left(K_{f}, K_{i}\right)\right| \phi_{i}\right\rangle,
$$

where the three-dimensional LF current operator, acting on the valence wave functions, is defined as follows

$$
j^{\mu}\left(K_{f}, K_{i}\right):=\bar{\Pi}\left(K_{f}\right) \mathcal{J}^{\mu}(Q) \Pi\left(K_{i}\right) .
$$

Using Eqs. (42) and (43), one can put in evidence the dependence of the LF current operator upon the effective interaction $W(K)$, viz.,

$$
\begin{aligned}
j^{\mu}\left(K_{f}, K_{i}\right):= & \bar{\Pi}_{0}\left(K_{f}\right)\left[1+W\left(K_{f}\right) \Delta_{0}\left(K_{f}\right)\right] \\
& \times \mathcal{J}^{\mu}(Q)\left[1+\Delta_{0}\left(K_{i}\right) W\left(K_{i}\right)\right] \Pi_{0}\left(K_{i}\right) .
\end{aligned}
$$

It is worth noting that Eq. (59) generalizes to scattering states the expression for the three-dimensional current already derived in Ref. [10] for bound states. It should be pointed out that $\mathcal{J}_{\mu}$ is Poincaré covariant [cf. Eq. (6) for the Lorentz covariance]. Since all the matrix elements of the left-hand side are properly related through the Lorentz 
transformations, the investigation of the covariance properties of the operator $j_{\mu}$, within the full theory, does not represent a stringent question in view of the equality in Eq. (57). But, given the following development of the truncated approach, where the full covariance is broken, it is important to investigate the transformation properties of $j_{\mu}$. As discussed elsewhere [27], we can anticipate that the covariance with respect to the kinematical transformations can be demonstrated after introducing new factors in the vertical bar operation (cf. also the factor $\hat{\Omega}$ in Ref. [11]).

In what follows, our task is to find a suitable definition of the LF charge operator that allows one to write the fourdivergence of the LF current operator in terms of the inverse of the Green's functions $g^{-1}\left(K_{f}\right)$ and $g^{-1}\left(K_{i}\right)$. Such an investigation is fundamental to obtain the WTI for the truncated LF current (see the next section). It should be pointed out that a similar analysis has been already performed for bosonic systems in [16].

In order to find the LF charge operator, the fourdimensional divergence of the LF current can be written as follows by using Eqs. (8) and (58)

$$
Q_{\mu} j^{\mu}\left(K_{f}, K_{i}\right)=\bar{\Pi}\left(K_{f}\right)\left[G^{-1}\left(K_{f}\right) \hat{e}-\hat{e} G^{-1}\left(K_{i}\right)\right] \Pi\left(K_{i}\right),
$$

with $\hat{e}=\hat{e}_{1}+\hat{e}_{2}$.

By applying Eqs. (B3) and (B4) of Appendix B, one gets

$$
\begin{aligned}
Q_{\mu} j^{\mu}\left(K_{f}, K_{i}\right)= & g^{-1}\left(K_{f}\right) \mid \bar{G}_{0}\left(K_{f}\right) G_{0}^{-1}\left(K_{f}\right) \hat{e} \Pi\left(K_{i}\right) \\
& -\bar{\Pi}\left(K_{f}\right) \hat{e} G_{0}^{-1}\left(K_{i}\right) \bar{G}_{0}\left(K_{i}\right) \mid g^{-1}\left(K_{i}\right) \\
= & g^{-1}\left(K_{f}\right) \hat{\mathcal{Q}}_{\mathrm{LF}}^{L}-\hat{\mathcal{Q}}_{\mathrm{LF}}^{R} g^{-1}\left(K_{i}\right)
\end{aligned}
$$

where the left and right LF charge operators have been introduced. Such operators are defined as follows

$$
\begin{aligned}
\hat{\mathcal{Q}}_{\mathrm{LF}}^{L} & =\mid \bar{G}_{0}\left(K_{f}\right) G_{0}^{-1}\left(K_{f}\right) \hat{e} \Pi\left(K_{i}\right) \\
& =\mid \bar{G}_{0}\left(K_{f}\right) G_{0}^{-1}\left(K_{f}\right) \hat{e} \Pi_{0}\left(K_{i}\right) \\
\hat{\mathcal{Q}}_{\mathrm{LF}}^{R} & =\bar{\Pi}\left(K_{f}\right) \hat{e} G_{0}^{-1}\left(K_{i}\right) \bar{G}_{0}\left(K_{i}\right) \mid \\
& =\bar{\Pi}_{0}\left(K_{f}\right) \hat{e} G_{0}^{-1}\left(K_{i}\right) \bar{G}_{0}\left(K_{i}\right) \mid
\end{aligned}
$$

where Eqs. (A3), (A4), and (32) have been used, as well as the absence of a Dirac structure in the operator $\hat{e}$. It is very important to note that the LF charge operators are noninteracting operators acting on the two-fermion spinor space. From Eqs. (A4) and (A5), we can obtain the explicit expression for the left LF charge operator for particle 1

$$
\hat{\mathcal{Q}}_{1 \mathrm{LF}}^{L}=\Lambda_{+}\left(\hat{k}_{1 \mathrm{on}}\right) \frac{m_{1}}{\hat{k}_{1}^{+}} \gamma_{1}^{+} \hat{e}_{1 \mathrm{LF}} \Lambda_{+}\left(\hat{k}_{1 \mathrm{on}}\right) \Lambda_{+}\left(\hat{k}_{2 \mathrm{on}}\right),
$$

where the notation $\hat{e}_{1 \mathrm{LF}}$ indicates the three-dimensional LF counterpart of the operator $\hat{e}$, Eq. (9), with matrix elements given by

$$
\begin{aligned}
\left\langle k_{1}^{\prime+}, \vec{k}_{1 \perp}^{\prime}\left|\hat{e}_{1 \mathrm{LF}}\right| k_{1}^{+}, \vec{k}_{1 j \perp}\right\rangle:= & e_{1} \delta\left(k_{1}^{\prime+}-k_{1}^{+}-Q^{+}\right) \\
& \times \delta^{2}\left(\vec{k}_{1 \perp}^{\prime}-\vec{k}_{1 \perp}-\vec{Q}_{\perp}\right) .
\end{aligned}
$$

The corresponding right operator is

$$
\hat{\mathcal{Q}}_{1 \mathrm{LF}}^{R}=\Lambda_{+}\left(\hat{k}_{1 \mathrm{on}}\right) \hat{e}_{1 \mathrm{LF}} \frac{m_{1}}{\hat{k}_{1}^{+}} \gamma_{1}^{+} \Lambda_{+}\left(\hat{k}_{1 \mathrm{on}}\right) \Lambda_{+}\left(\hat{k}_{2 \mathrm{on}}\right) .
$$

The operator $\gamma^{+} m / k^{+}$when sandwiched between LF spinors gives the normalization condition. Let us remind the reader that such an operator is the one particle free charge operator of our model, since we do not include fermion self-energy. This also indicates the ingredients that ought to be considered when the full problem with self-energy insertions is aimed. We will not discuss further this issue here, which is left for a future study.

From Eq. (61), current conservation straightforwardly follows by taking the matrix elements between threedimensional interacting states that are solutions of the wave equation (45) and noting that the left and right charge operators do not contain any $l \varepsilon$ dependence.

By multiplying both the left and right hand sides of Eq. (61) by $g\left(K_{f}\right)$ and $g\left(K_{i}\right)$, respectively, one gets

$$
Q_{\mu} g\left(K_{f}\right) j^{\mu}\left(K_{f}, K_{i}\right) g\left(K_{i}\right)=\hat{\mathcal{Q}}_{\mathrm{LF}}^{L} g\left(K_{i}\right)-g\left(K_{f}\right) \hat{\mathcal{Q}}_{\mathrm{LF}}^{R},
$$

which corresponds to the LF projection of the five-point function with instantaneous terms cut from the external fermion legs.

In conclusion the three-dimensional LF current given by Eq. (58) acts on the LF valence wave functions and fulfills WTI [see Eq. (67)].

\section{WARD-TAKAHASHI IDENTITY FOR THE TRUNCATED LF CURRENT}

In this section, to obtain a workable approximation for the LF current operator that still fulfills WTI, we will analyze the consequences of a proper truncation of the QP expansion in the formal solution [Eq. (33)] of Eq. (30). As already anticipated in the previous section, the LF charges and the general form for the LF WTI [cf Eq. (61)] will represent our fundamental ingredients. To simplify notations, we will not show explicitly the parametric dependence on total momentum $K$ in the operators. Therefore, it is understood that the operators on the left of the charge operator depend upon $K_{f}$, while the ones on the right depend upon $K_{i}$.

A naive substitution of $W$ in the current operator (59), by the truncated quasipotential expansion

$$
W^{(n)}=\sum_{i=1}^{n} W_{i} \quad \text { with } W_{i}=V\left[\Delta_{0} V\right]^{i-1}=\left[V \Delta_{0}\right]^{i-1} V,
$$

namely, by the truncated iterative solution of Eq. (30), does 
not lead to a conserved three-dimensional current. The same problem has been met and solved for two-boson systems in Ref. [16]. In this section we will follow the same procedure, but applied to the case of two-fermion systems. In the next section an actual application for the Yukawa model will be illustrated.

Let us first consider the conserved current, for the case $n=0$, i.e., the current without interaction. In this case one has

$$
j^{c(0) \mu}:=\bar{\Pi}_{0} \mathcal{J}_{0}^{\mu} \Pi_{0}=g_{0}^{-1}\left|\bar{G}_{0} \mathcal{J}_{0}^{\mu} \bar{G}_{0}\right| g_{0}^{-1} .
$$

From Eqs. (11), (62), (63), and (69), WTI for $n=0$ reads

$$
\begin{aligned}
Q^{\mu} j_{\mu}^{c(0)} & =g_{0}^{-1}\left|\bar{G}_{0} G_{0}^{-1} \hat{e} \Pi_{0}-\bar{\Pi}_{0} \hat{e} G_{0}^{-1} \bar{G}_{0}\right| g_{0}^{-1} \\
& =g_{0}^{-1} \hat{\mathcal{Q}}_{\mathrm{LF}}^{L}-\hat{\mathcal{Q}}_{\mathrm{LF}}^{R} g_{0}^{-1} .
\end{aligned}
$$

The matrix elements of Eq. (70) should be taken between solutions of $g_{0}^{-1}\left|\phi_{0}\right\rangle=0$.

The matrix elements of $j_{\mu}^{c(0)}$ between free particle states, as obtained explicitly in Appendix $\mathrm{C}$ together with the corresponding WTI, are given by

$$
\begin{aligned}
\left\langle k_{1}^{\prime+} \vec{k}_{1 \perp}^{\prime}\left|j^{c(0) \mu}\right| k_{1}^{+} \vec{k}_{1 \perp}\right\rangle= & -i \theta\left(k_{1}^{+}\right) \theta\left(k_{1}^{\prime+}\right) \theta\left(K_{i}^{+}-k_{1}^{+}\right) \theta\left(K_{f}^{+}-k_{1}^{\prime+}\right) \Lambda_{+}\left(k_{1 \mathrm{on}}^{\prime}\right) \gamma_{1}^{\mu} \Lambda_{+}\left(k_{1 \mathrm{on}}\right) \Lambda_{+}\left(\left(K_{i}-k_{1}\right)_{\mathrm{on}}\right) \\
& \times \frac{K_{i}^{+}-k_{1}^{+}}{2 m_{2}}\left\langle k_{1}^{\prime+}, \vec{k}_{1 \perp}^{\prime}\left|\hat{e}_{1, \mathrm{LF}}\right| k_{1}^{+} \vec{k}_{1 \perp}\right\rangle+1 \leftrightarrow 2 .
\end{aligned}
$$

From Eq. (59) and cutting at the first order the effective interaction (note that $W^{(1)}=V$ ) one has

$$
j^{(1) \mu}=j^{c(1) \mu}+\mathcal{O}\left(V^{2}\right)+\mathcal{O}\left(V^{3}\right)
$$

where the first-order contribution is given by

$$
\begin{aligned}
j^{c(1) \mu} & =\bar{\Pi}_{0}\left[\mathcal{J}^{\mu}+V \Delta_{0} \mathcal{J}_{0}^{\mu}+\mathcal{J}_{0}^{\mu} \Delta_{0} V\right] \Pi_{0} \\
& =j^{c(0) \mu}+\bar{\Pi}_{0}\left[\mathcal{J}_{I}^{\mu}+V \Delta_{0} \mathcal{J}_{0}^{\mu}+\mathcal{J}_{0}^{\mu} \Delta_{0} V\right] \Pi_{0} .
\end{aligned}
$$

Only $j^{c(1) \mu}$ is a conserved current operator in the corresponding valence sector. Indeed, as shown in detail in Appendix D, one obtains the following WTI

$$
Q^{\mu} j_{\mu}^{c(1)}=g_{1}{ }^{-1} \hat{\mathcal{Q}}_{\mathrm{LF}}^{L}-\hat{\mathcal{Q}}_{\mathrm{LF}}^{R} g_{1}{ }^{-1},
$$

where $g_{1}^{-1}=g_{0}{ }^{-1}-w^{(1)}$ and $w^{(1)}=\bar{\Pi}_{0} V \Pi_{0}$.

The matrix elements of Eq. (74) should be taken between solutions of $g_{1}^{-1}\left|\phi_{1}\right\rangle=0$. In Sec. VII, we will discuss in detail the first-order LF current for the Yukawa model in ladder approximation, and we will show that it is essential to include the instantaneous terms, coming from $\Delta_{0}$ to obtain WTI for $n=1$.

For the general case, where the interaction appears up to $n \geq 1$ times in the LF current operator, we write

$$
\begin{aligned}
j^{c(n) \mu}:= & \bar{\Pi}_{0}\left[\mathcal{J}^{\mu}+W_{n} \Delta_{0} \mathcal{J}_{0}^{\mu}+\mathcal{J}_{0}^{\mu} \Delta_{0} W_{n}+\sum_{i=1}^{n-1}\left(W_{i} \Delta_{0} \mathcal{J}_{0}^{\mu} \Delta_{0} W_{n-i}+W_{i} \Delta_{0} \mathcal{J}^{\mu}+\mathcal{J}^{\mu} \Delta_{0} W_{i}\right)\right. \\
& \left.+\sum_{j=2}^{n-1} \sum_{i=1}^{j-1} W_{i} \Delta_{0} \mathcal{J}^{\mu} \Delta_{0} W_{j-i}\right] \Pi_{0} . \\
= & j^{c(n-1) \mu}+\bar{\Pi}_{0}\left[\sum_{i=0}^{n} W_{i} \Delta_{0} \mathcal{J}_{0}^{\mu} \Delta_{0} W_{n-i}+\sum_{i=0}^{n-1} W_{i} \Delta_{0} \mathcal{J}_{I}^{\mu} \Delta_{0} W_{n-1-i}\right] \Pi_{0},
\end{aligned}
$$

where it has been formally defined $W_{0} \Delta_{0}=\Delta_{0} W_{0}=1$. It is worth noting that Eq. (75) contains power of the interaction up to the $n$th order, since $\mathcal{J}_{0}^{\mu}$ is $O\left(V^{0}\right)$ and $\mathcal{J}_{I}^{\mu}$ is $O\left(V^{1}\right)$.

As demonstrated by induction in Appendix E, this truncated current operator satisfies a WTI given by

$$
Q^{\mu} j_{\mu}^{c(n)}=g_{n}{ }^{-1} \hat{\mathcal{Q}}_{\mathrm{LF}}^{L}-\hat{\mathcal{Q}}_{\mathrm{LF}}^{R} g_{n}{ }^{-1},
$$

where $g_{n}{ }^{-1}=g_{0}{ }^{-1}-w^{(n)}$ and $w^{(n)}$ is the truncated effective interaction, viz.,

$$
w^{(n)}=\bar{\Pi}_{0} W^{(n)} \Pi_{0}=\sum_{i=1}^{n} \bar{\Pi}_{0} W_{i} \Pi_{0} .
$$

The matrix elements of Eq. (76) should be taken between solutions of $g_{n}^{-1}\left|\phi_{n}\right\rangle=0$.

Thus, we conclude that the LF electromagnetic current operator $j_{\mu}^{c(n)}$ is conserved at any given order $n$ of the QP expansion. In the limit $n \rightarrow \infty$ the truncated conserved current becomes the full current operator of the model, if the QP expansion converges (for numerical studies of the convergence see, e.g., $[9,25])$. Finally, the effects of the truncation in the QP expansion, and correspondingly in the Fock-space expansion, on the transformation properties under the Poincare group will be investigated elsewhere [27].

It should be pointed out that the last line of Eq. (75) suggests a possible diagrammatic representation of the 
current (cf. the next section for the first-order case in a simple example), through the observation that the contribution to the quasipotential at a $p$ th order, $W_{p}$, can be decomposed in $W_{p}=W_{i} \Delta_{0} W_{p-i}$ for $p \geq i \geq 0$ [see, Eq. (68)]. Indeed one can construct a picture of the contribution between square brackets in the last line of Eq. (75), by starting with all the possible decompositions for $W_{n}$ and $W_{n-1}$, then breaking the subtracted propagation represented by $\Delta_{0}$ and inserting the current $\mathcal{J}_{0}$ and $\mathcal{J}_{I}$, respectively, i.e., $\Delta_{0} \rightarrow \Delta_{0} \mathcal{J}_{0(I)} \Delta_{0}$.

As shown in detail in the example of the next section, the subtracted Green's function $\Delta_{0}$ eliminates the presence of disconnected diagrams, due to the global, free-propagation of the two-fermions between the photon absorption and the exchange of a boson from the interaction. At the same time, it allows for the photon being absorbed (i) instantaneously (in LF time), or (ii) by higher Fockstate components, including a particle-antiparticle pair. In particular, one can easily see that the disconnected diagrams generated from the gauging on the external legs, i.e., diagrams that are already taken into account through the interacting LF wave function, are eliminated by the presence of the subtraction in $\Delta_{0}$, avoiding double counting.

\section{LF CURRENT AND WARD-TAKAHASHI IDENTITY IN THE LADDER YUKAWA MODEL AT THE FIRST-ORDER}

In this section we explicitly derive the current operator $j^{c(1) \mu}$, see Eq. (73), for a two-fermion system interacting through chargeless bosons, within the Yukawa model in ladder approximation. This will illustrate in an actual case how our procedure works to obtain the conserved current operator at the first nontrivial order, namely $n=1$. The Yukawa model is defined by the following interacting Lagrangian (with no derivative coupling):

$$
\mathcal{L}_{I}=g \bar{\psi}_{1} \Gamma_{1}^{\alpha} \psi_{1} \sigma_{\alpha}+g \bar{\psi}_{2} \Gamma_{2}^{\alpha} \psi_{2} \sigma_{\alpha},
$$

where $g$ is the coupling constant (that should not be confused with the LF Green's function $g(k)), \psi_{1}$ and $\psi_{2}$ are fermionic fields, the corresponding particles have masses $m_{1}$ and $m_{2}$, and charges $e_{1}$ and $e_{2}$, respectively. The field $\sigma_{\alpha}$ corresponds to a chargeless boson with mass $\mu$. The index $\alpha$ represents Lorentz components in the case of a vector or tensor field. The vertex $\Gamma_{j}^{\alpha}$ is defined in the spinor space. The four-dimensional interaction, $V$ is given by

$$
V=i g^{2} \frac{\Gamma_{1}^{\alpha} \otimes \Gamma_{2 \alpha}}{\left(\hat{p}_{1}-\hat{p}_{2}\right)^{2}-\mu^{2}+i \varepsilon}
$$

where the symbol $\otimes$ takes distinct the vertices corresponding to the fermions 1 and 2 .

We choose this simple example to show that an interacting LF two-body current can be generated even starting from a four-dimensional free current, $\mathcal{J}_{0}^{\mu}$. It should be pointed out that in ladder BS approximation the consistent

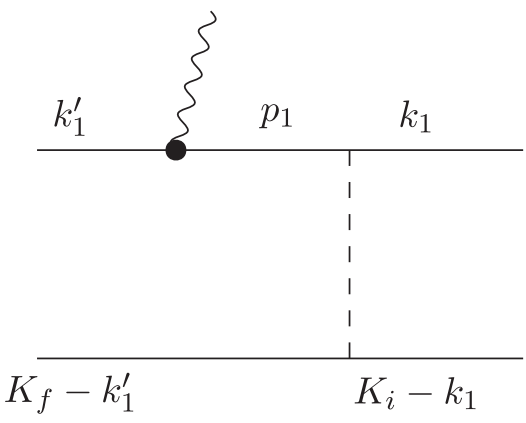

FIG. 1. Diagrammatic representation of the matrix elements of the four-dimensional operator $\bar{G}_{0} \mathcal{J}_{0}^{\mu} G_{0} V \bar{G}_{0}$, [cf. Eqs. (F1) and (F2)]. Dashed line: four-dimensional interaction $V$. The full dot represents the free current.

four current is the free one, i.e., $\mathcal{J}^{\mu} \rightarrow \mathcal{J}_{0}^{\mu}$, and the fourdimensional WTI is fulfilled because the commutator $[\hat{e}, V]$ trivially vanishes. Such a cancellation is due to the fact that the exchanged momentum in the potential just depends on the spectator particle momentum, that remains unchanged in the transition from the initial state to the final one (cf. Ref. [1] and Fig. 1). Since we need all the four components of $j^{c(1) \mu}$ to construct the WTI, Eq. (74), in what follows we explicitly evaluate all the components of the truncated em current, by projecting the fourdimensional current onto the LF hypersurface (i.e., by integrating on $k^{-}$).

Let us start with the matrix elements of the free current operator, given by

$$
\begin{aligned}
\left\langle k_{1}\left|\mathcal{J}_{0}^{\mu}(Q)\right| p_{1}\right\rangle= & -2 \pi e_{1} \gamma_{1}^{\mu} \delta^{4}\left(k_{1}-p_{1}-Q\right) \\
& \left.\times\left[\left(\not K_{f}-\not k_{1}\right)-m_{2}\right)\right] \\
& +\left[1 \rightarrow 2, k_{1} \rightarrow K_{f}-k_{1}, p_{1} \rightarrow K_{i}-p_{1}\right],
\end{aligned}
$$

where $Q^{\mu}=K_{f}^{\mu}-K_{i}^{\mu}$. The factor $(-2 \pi)$ is introduced in the current operator to make it compatible with the free Green's function, see Eq. (3).

The first-order current operator for the interacting twofermion system in this example is given by [cf. Eq. (73)],

$$
j^{c(1) \mu}=j^{c(0) \mu}+\bar{\Pi}_{0}\left[V \Delta_{0} \mathcal{J}_{0}^{\mu}+\mathcal{J}_{0}^{\mu} \Delta_{0} V\right] \Pi_{0},
$$

where the zero-order LF current operator is the LF free current, see, Eqs. (69) and (71).

The contribution of the interaction to the LF current operator in lowest order comes from two-body irreducible amplitudes given by the second term in the rhs of Eq. (73) with $\mathcal{J}^{\mu} \equiv \mathcal{J}_{0}^{\mu}$, since we are adopting the ladder approximation. Using Eq. (31) for $\Delta_{0}$ we write that

$$
\begin{aligned}
j^{c(1) \mu}-j^{c(0) \mu}= & \bar{\Pi}_{0} V G_{0} \mathcal{J}_{0}^{\mu} \Pi_{0}-w^{(1)} g_{0} j^{c(0) \mu} \\
& +\bar{\Pi}_{0} \mathcal{J}_{0}^{\mu} G_{0} V \Pi_{0}-j^{c(0) \mu} g_{0} w^{(1)}
\end{aligned}
$$


where $w^{(1)}=\bar{\Pi}_{0} V \Pi_{0}$ is the three-dimensional effective interaction, with matrix elements for a total momentum $K$ given by (see Appendix C in [10])

$$
\begin{aligned}
\left\langle k_{1}^{\prime+} \vec{k}_{1 \perp}^{\prime}\left|w^{(1)}(K)\right| k_{1}^{+} \vec{k}_{1 \perp}\right\rangle= & i(i g)^{2} \theta\left(k_{1}^{\prime+}\right) \theta\left(k_{1}^{+}\right) \theta\left(K^{+}-k_{1}^{\prime+}\right) \theta\left(K^{+}-k_{1}^{+}\right)\left[\frac{\theta\left(k_{1}^{\prime+}-k_{1}^{+}\right)}{\left(k_{1}^{\prime+}-k_{1}^{+}\right)\left(K^{-}-k_{1 \mathrm{on}}^{-}-k_{2 \mathrm{on}}^{\prime-}-k_{\sigma \mathrm{on}}+i \varepsilon\right)}\right. \\
& \left.+\frac{\theta\left(k_{1}^{+}-k_{1}^{\prime+}\right)}{\left(k_{1}^{+}-k_{1}^{\prime+}\right)\left(K^{-}-k_{1 \mathrm{on}}^{\prime-}-k_{2 \mathrm{on}}^{-}+k_{\sigma \mathrm{on}}+i \varepsilon\right)}\right] \Lambda_{+}\left(k_{1 \mathrm{on}}^{\prime}\right) \Gamma_{1}^{\alpha} \Lambda_{+}\left(k_{1 \mathrm{on}}\right) \Lambda_{+}\left(k_{2 \mathrm{on}}^{\prime}\right) \Gamma_{2 \alpha} \Lambda_{+}\left(k_{2 \mathrm{on}}\right),
\end{aligned}
$$

where $k_{2}=K-k_{1}$,

$$
\begin{gathered}
k_{\text {lon }}^{-}=\frac{\vec{k}_{1 \perp}^{2}+m_{1}^{2}}{k_{1}^{+}} \quad k_{\text {lon }}^{\prime-}=\frac{\vec{k}_{1 \perp}^{2}+m_{1}^{2}}{k_{1}^{\prime+}} \\
k_{\sigma \text { on }}^{-}=\frac{\left(\vec{k}_{1 \perp}^{\prime}-\vec{k}_{1 \perp}\right)^{2}+\mu^{2}}{\left(k_{1}^{\prime+}-k_{1}^{+}\right)}
\end{gathered}
$$

and the quantities corresponding to fermion 2 easily follows. As shown in Appendix F, both the first term and the third one in the rhs of Eq. (82) contain a LF two-body reducible part (already taken into account when $j^{c(0) \mu}$ is applied to the valence wave function), that is canceled by the second and the fourth ones, respectively. In particular (see, Ref. [10]), the LF two-body reducible contributions are generated by the effective interaction used to obtain the valence wave function at the first order in the quasipotential expansion of the ladder BS equation. The essential role played by the reducible terms was stressed in [28], in a calculation of higher Fock states contributions to the generalized parton distribution of pion.

The difference with the boson case, that was recently studied [16], comes from the instantaneous terms present in the quasipotential expansion of the fermionic current operator, as we will show in detail.
In the following we report the matrix elements of the first-order current operator relevant for the em processes in the spacelike region, as obtained in Appendix F.

\section{A. The term $\bar{\Pi}_{0} \mathcal{J}_{0}^{\mu}(Q) G_{0} V \Pi_{0}$}

In Fig. 2, it is diagrammatically illustrated the third term in the rhs of Eq. (82), $g_{0}^{-1}\left|\bar{G}_{0} \mathcal{J}_{0}^{\mu}(Q) G_{0} V \bar{G}_{0}\right| g_{0}^{-1}$. It is worthwhile to note that this term contains the relevant pair production contribution, for $Q^{+}>0$ (cf. diagram (b) in Fig. 2). Differently, in the first term, $g_{0}^{-1}\left|\bar{G}_{0} V G_{0} \mathcal{J}_{0}^{\mu}(Q) \bar{G}_{0}\right| g_{0}^{-1}$, there is no contribution from the pair production by the virtual photon, since the conservation of the plus momentum component always requires a positive plus component of the intermediate fermion momentum and then the initial state cannot contain a photon and a pair (cf. Fig. 3).

Performing analytical integrations over $k_{1}^{-}$and $k_{1}^{\prime}$ by Cauchy's theorem with the conditions $K_{i}^{+}>0$ and $Q^{+} \geq$ 0 (see Appendix F), we get the six contributions that appear in Fig. 2. We observe that the limited number of LF timeordered diagrams are essentially a consequence of the trivial vacuum structure, due to the conservation of the total positive plus momentum. Now, let us discuss in detail the irreducible diagrams of Fig. 2.

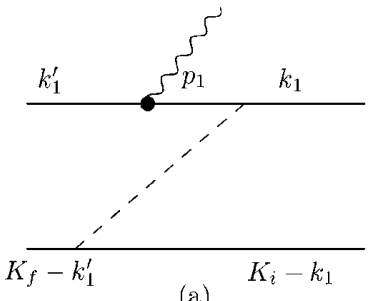

(a)

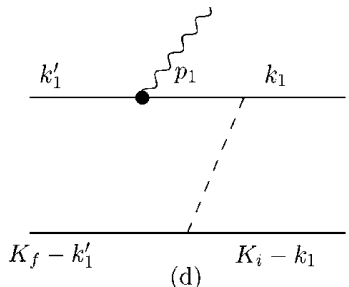

(d)

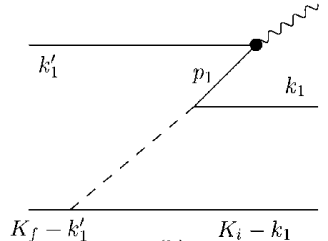

(b)

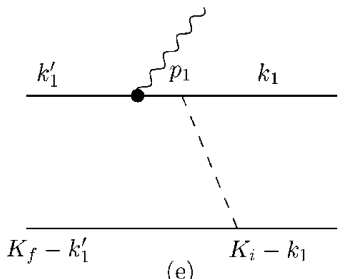

(e)
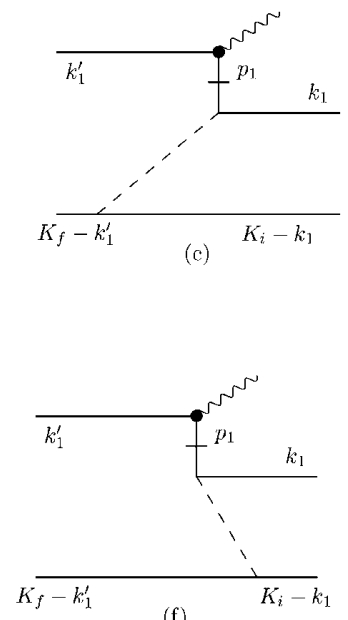

(f)

FIG. 2. LF time-ordered diagrams representing the matrix element of the current operator obtained from $\bar{\Pi}_{0} \mathcal{J}_{0}^{\mu}(Q) G_{0} V \Pi \Pi_{0}$, i.e., the third term in the rhs of Eq. (82). The reducible diagrams, (d) and (e), are canceled by $j^{c(0) \mu} g_{0} w^{(1)}$. 

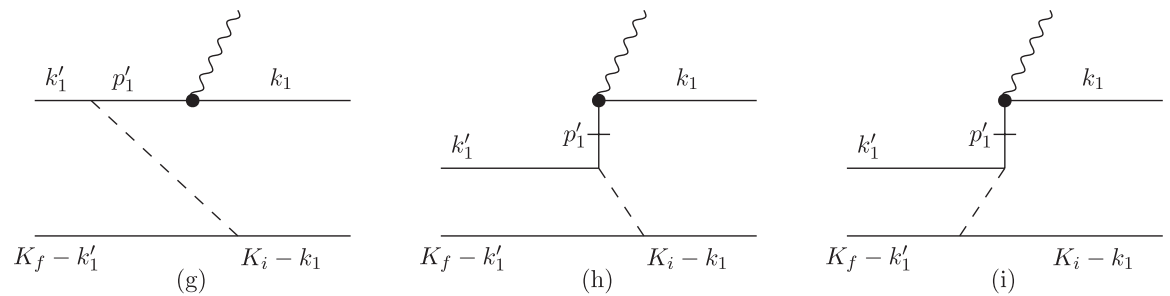

FIG. 3. LF time-ordered diagrams representing the matrix element of the current operator obtained from $\bar{\Pi}_{0} V G_{0} \mathcal{J}_{0}^{\mu}(Q) \Pi_{0}$, i.e., the first term in the rhs of Eq. (82). The reducible diagrams, canceled by $w^{(1)} j^{c(0) \mu} g_{0}$, are not shown.

Diagram (a) gives a two-body current with intermediate three-body virtual state propagations before and after the photon absorption, in the kinematical range $p_{1}^{+}=k_{1}^{\prime+}-$ $Q^{+} \geq 0$ and $k_{1}^{+}>\left(k_{1}^{\prime+}-Q^{+}\right)$. Diagram (b) represents the contribution to the current of the virtual photon decay in a pair. The kinematical condition in this case is $p_{1}^{+}=k_{1}^{\prime+}-$ $Q^{+}<0$ (note that $-Q^{+}<p_{1}^{+}<0$ ). Diagram (c) represents a contribution from the instantaneous term of the fermion propagator, which is nonvanishing in two kine- matical regions: (i) $p_{1}^{+}=k_{1}^{\prime+}-Q^{+} \geq 0$ and $k_{1}^{+}>\left(k_{1}^{\prime+}-\right.$ $Q^{+}$), (ii) $p_{1}^{+}=k_{1}^{\prime+}-Q^{+}<0$. Diagrams (d) and (e) are two-body reducible terms which are canceled by $j^{c(0) \mu} g_{0} w^{(1)}$ in Eq. (82). Diagram (f) represents a contribution of the instantaneous term for $p_{1}^{+}=k_{1}^{\prime+}-Q^{+}>$ $k_{1}^{+}>0$.

Defining $\Theta=\theta\left(k_{1}^{+}\right) \theta\left(k_{1}^{+\prime}\right) \theta\left(K_{i}^{+}-k_{1}^{+}\right) \theta\left(K_{f}^{+}-k_{1}^{+\prime}\right)$, diagram (a) is given by [see, Eq. (F7)]

$$
\begin{aligned}
\left\langle k_{1}^{\prime+} \vec{k}_{1 \perp}^{\prime}\left|j^{c(1) \mu}\right| k_{1}^{+} \vec{k}_{1 \perp}\right\rangle_{(a)}= & i e_{1}\left(2 m_{1}\right)(i g)^{2} \Theta \frac{\theta\left(k_{1}^{+}-p_{1}^{+}\right)}{\left(k_{1}^{+}-p_{1}^{+}\right) p_{1}^{+}} \frac{\theta\left(p_{1}^{+}\right)}{\Delta_{f}^{(1 a)-} \Delta_{i}^{(1 a)-}} \\
& \times \Lambda_{+}\left(k_{1 \mathrm{on}}^{\prime}\right) \gamma_{1}^{\mu} \Lambda_{+}\left(p_{1 \mathrm{on}}\right) \Gamma_{1}^{\alpha} \Lambda_{+}\left(k_{1 \mathrm{on}}\right) \Lambda_{+}\left(\left(K_{f}-k_{1}^{\prime}\right)_{\mathrm{on}}\right) \Gamma_{2 \alpha} \Lambda_{+}\left(\left(K_{i}-k_{1}\right)_{\mathrm{on}}\right) .
\end{aligned}
$$

The four vectors $\Delta_{f}^{(1 a)}$ and $\Delta_{i}^{(1 a)}$ are the following combinations of the four momenta

$$
\Delta_{f}^{(1 a)}=K_{f}-k_{\text {lon }}^{\prime}-\left(K_{i}-k_{1}\right)_{\mathrm{on}}-\left(k_{1}-p_{1}\right)_{\mathrm{on}}+i \varepsilon \quad \Delta_{i}^{(1 a)}=K_{i}-p_{\text {1on }}-\left(K_{i}-k_{1}\right)_{\mathrm{on}}-\left(k_{1}-p_{1}\right)_{\mathrm{on}}+i \varepsilon .
$$

The minus components yield the three-body (two fermions and the exchanged boson) global propagation in the final and initial states, respectively.

The matrix element of the current operator (b) (virtual photon decay in a pair) is [see, Eq. (F13)]

$$
\begin{aligned}
\left\langle k_{1}^{\prime+} \vec{k}_{1 \perp}^{\prime}\left|j^{c(1)_{\mu}}\right| k_{1}^{+} \vec{k}_{1 \perp}\right\rangle_{(b)}= & -i e_{1}\left(2 m_{1}\right)(i g)^{2} \Theta \frac{\theta\left(-p_{1}^{+}\right)}{p_{1}^{+}} \frac{\theta\left(k_{1}^{+}-p_{1}^{+}\right)}{\left(k_{1}^{+}-p_{1}^{+}\right) \Delta_{f}^{(1 a)-} \Delta_{\gamma}^{(1 b)-}} \Lambda_{+}\left(k_{1 \mathrm{on}}^{\prime}\right) \gamma_{1}^{\mu} \Lambda_{+}\left(p_{1 \mathrm{on}}\right) \Gamma_{1}^{\alpha} \Lambda_{+}\left(k_{\text {lon }}\right) \\
& \times \Lambda_{+}\left(\left(K_{f}-k_{1}^{\prime}\right)_{\mathrm{on}}\right) \Gamma_{2 \alpha} \Lambda_{+}\left(\left(K_{i}-k_{1}\right)_{\mathrm{on}}\right),
\end{aligned}
$$

where the combination $\Delta_{\gamma}^{(1 b)}$ of four momenta is

$$
\Delta_{\gamma}^{(1 b)}=Q-k_{\text {lon }}^{\prime}+p_{\text {lon }}+i \varepsilon .
$$

The instantaneous term (c) [see, Eqs. (F9) and (F15) in Appendix F15] can be expressed as

$$
\begin{aligned}
\left\langle k_{1}^{\prime+} \vec{k}_{1 \perp}^{\prime}\left|j^{c(1) \mu}\right| k_{1}^{+} \vec{k}_{1 \perp}\right\rangle_{(c)}= & \frac{i}{2} e_{1}(i g)^{2} \Theta \frac{1}{p_{1}^{+}} \frac{\theta\left(k_{1}^{+}-p_{1}^{+}\right)}{\left(k_{1}^{+}-p_{1}^{+}\right) \Delta_{f}^{(1 a)-}} \Lambda_{+}\left(k_{1 \mathrm{on}}^{\prime}\right) \gamma_{1}^{\mu} \gamma_{1}^{+} \Gamma_{1}^{\alpha} \Lambda_{+}\left(k_{1 \mathrm{on}}\right) \Lambda_{+}\left(\left(K_{f}-k_{1}^{\prime}\right)_{\mathrm{on}}\right) \\
& \times \Gamma_{2 \alpha} \Lambda_{+}\left(\left(K_{i}-k_{1}\right)_{\mathrm{on}}\right) .
\end{aligned}
$$

Finally the contribution from the instantaneous term for $k_{1}^{\prime+}-Q^{+}>k_{1}^{+}$, represented by diagram (f), is [see, Eq. (F11)]

$$
\begin{aligned}
\left\langle k_{1}^{\prime+} \vec{k}_{1 \perp}^{\prime}\left|j^{c(1) \mu}\right| k_{1}^{+} \vec{k}_{1 \perp}\right\rangle_{(f)}= & \frac{i}{2} e_{1}(i g)^{2} \Theta \frac{\theta\left(p_{1}^{+}\right)}{p_{1}^{+}} \frac{\theta\left(p_{1}^{+}-k_{1}^{+}\right)}{\left(p_{1}^{+}-k_{1}^{+}\right) \Delta_{i}^{(1 f)-}} \\
& \times \Lambda_{+}\left(k_{1 \mathrm{on}}^{\prime}\right) \gamma_{1}^{\mu} \gamma_{1}^{+} \Gamma_{1}^{\alpha} \Lambda_{+}\left(k_{1 \mathrm{on}}\right) \Lambda_{+}\left(\left(K_{f}-k_{1}^{\prime}\right)_{\mathrm{on}}\right) \Gamma_{2 \alpha} \Lambda_{+}\left(\left(K_{i}-k_{1}\right)_{\mathrm{on}}\right),
\end{aligned}
$$


where the denominator is the minus component of the following four momentum

$$
\Delta_{i}^{(1 f)}=K_{i}-k_{1 \mathrm{on}}-\left(K_{f}-k_{1}^{\prime}\right)_{\mathrm{on}}-\left(p_{1}-k_{1}\right)_{\mathrm{on}}
$$

and yields the intermediate propagation of the three-body system composed by two fermions and a boson.

\section{B. The term $\bar{\Pi}_{\mathbf{0}} V \boldsymbol{G}_{\mathbf{0}} \mathcal{J}_{\mathbf{0}}^{\boldsymbol{\mu}}(Q) \Pi_{\mathbf{0}}$}

Let us now discuss the first contribution in Eq. (82), $g_{0}^{-1}\left|\bar{G}_{0} V G_{0} \mathcal{J}_{0}^{\mu}(Q) \bar{G}_{0}\right| g_{0}^{-1}$, depicted by diagrams (g) to (i) in Fig. 3. It is worth noting that the pair contribution is not present due to the conservation of the plus momentum component. For the sake of simplicity, the two reducible diagrams, which are canceled by $w^{(1)} g_{0} j^{c(0) \mu}$ in Eq. (82) in an analogous way as we have already discussed for diagrams (e) and (d) in Fig. 2, are not shown in Fig. 3. As discussed in detail in Appendix F, by analytical integrations over $k_{1}^{-}$and $k_{1}^{\prime}$ by Cauchy's theorem one can express diagram (g) as ([see, Eq. (F21)]

$$
\begin{aligned}
\left\langle k_{1}^{\prime+} \vec{k}_{1 \perp}^{\prime}\left|j^{c(1) \mu}\right| k_{1}^{+} \vec{k}_{1 \perp}\right\rangle_{(g)}= & i e_{1}\left(2 m_{1}\right)(i g)^{2} \Theta \frac{\theta\left(k_{1}^{\prime+}-p_{1}^{\prime+}\right)}{\left(k_{1}^{\prime+}-p_{1}^{\prime+}\right) p_{1}^{\prime+} \Delta_{f}^{(1 g)-} \Delta_{i}^{(1 g)-}} \Lambda_{+}\left(k_{1 \mathrm{on}}^{\prime}\right) \Gamma_{1}^{\alpha} \Lambda_{+}\left(p_{1 \mathrm{on}}^{\prime}\right) \gamma_{1}^{\mu} \Lambda_{+}\left(k_{1 \mathrm{on}}\right) \\
& \times \Lambda_{+}\left(\left(K_{f}-k_{1}^{\prime}\right)_{\mathrm{on}}\right) \Gamma_{2 \alpha} \Lambda_{+}\left(\left(K_{i}-k_{1}\right)_{\mathrm{on}}\right)
\end{aligned}
$$

where the combinations $\Delta_{f}^{(1 g)}$ and $\Delta_{i}^{(1 g)}$ of four momenta are

$\Delta_{f}^{(1 g)}=K_{f}-p_{1 \mathrm{on}}^{\prime}-\left(K_{f}-k_{1}^{\prime}\right)_{\mathrm{on}}-\left(k_{1}^{\prime}-p_{1}^{\prime}\right)_{\mathrm{on}}+i \varepsilon \quad \Delta_{i}^{(1 g)}=K_{i}-k_{1 \mathrm{on}}-\left(K_{f}-k_{1}^{\prime}\right)_{\mathrm{on}}-\left(k_{1}^{\prime}-p_{1}^{\prime}\right)_{\mathrm{on}}+i \varepsilon=\Delta_{i}^{(1 f)}$,

since $k_{1}^{\prime}-p_{1}^{\prime}=p_{1}-k_{1}$.

The instantaneous diagram $(\mathrm{h})$ is given by [see, Eq. (F23)]

$$
\begin{aligned}
\left\langle k_{1}^{\prime+} \vec{k}_{1 \perp}^{\prime}\left|j^{c(1) \mu}\right| k_{1}^{+} \vec{k}_{1 \perp}\right\rangle_{(h)}= & \frac{i}{2} e_{1}(i g)^{2} \Theta \frac{\theta\left(k_{1}^{\prime+}-p_{1}^{\prime+}\right)}{\left(k_{1}^{\prime+}-p_{1}^{\prime+}\right) p_{1}^{\prime+} \Delta_{i}^{(1 g)-}} \Lambda_{+}\left(k_{1 \mathrm{on}}^{\prime}\right) \Gamma_{1}^{\alpha} \gamma_{1}^{+} \gamma_{1}^{\mu} \Lambda_{+}\left(k_{1 \mathrm{on}}\right) \Lambda_{+}\left(\left(K_{f}-k_{1}^{\prime}\right)_{\mathrm{on}}\right) \\
& \times \Gamma_{2 \alpha} \Lambda_{+}\left(\left(K_{i}-k_{1}\right)_{\mathrm{on}}\right)
\end{aligned}
$$

while the instantaneous contribution in the region $p_{1}^{\prime+}>k_{1}^{\prime+}>0$, illustrated by diagram (i) in Fig. 3, is [see, Eq. (F24)]

$$
\begin{aligned}
\left\langle k_{1}^{\prime+} \vec{k}_{1 \perp}^{\prime}\left|j^{c(1) \mu}\right| k_{1}^{+} \vec{k}_{1 \perp}\right\rangle_{(i)}= & \frac{i}{2} e_{1}(i g)^{2} \Theta \frac{\theta\left(p_{1}^{\prime+}-k_{1}^{\prime+}\right)}{\left(p_{1}^{\prime+}-k_{1}^{\prime+}\right) p_{1}^{\prime+} \Delta_{f}^{(1 i)-}} \Lambda_{+}\left(k_{1 \mathrm{on}}^{\prime}\right) \Gamma_{1}^{\alpha} \gamma_{1}^{+} \gamma_{1}^{\mu} \Lambda_{+}\left(k_{1 \mathrm{on}}\right) \Lambda_{+}\left(\left(K_{f}-k_{1}^{\prime}\right)_{\mathrm{on}}\right) \\
& \times \Gamma_{2 \alpha} \Lambda_{+}\left(\left(K_{i}-k_{1}\right)_{\mathrm{on}}\right)
\end{aligned}
$$

where the three-body denominator is the minus component of

$$
\Delta_{f}^{(1 i)}=K_{f}-k_{1 \mathrm{on}}^{\prime}-\left(K_{i}-k_{1}\right)_{\mathrm{on}}-\left(p_{1}^{\prime}-k_{1}^{\prime}\right)_{\mathrm{on}}=\Delta_{f}^{(1 a)} .
$$

Closing the discussion on the first-order current, we refer the reader to Appendix $G$ for an explicit check of the current conservation. The detailed derivation of current conservation allows a deeper understanding of the explicit effects of the formal manipulations used to obtain a conserved LF operator, consistent with the effective interaction at each given order. This calculation also shows the essential role played by the instantaneous terms in the cancellation of terms from the two-body current (a) and (g), that otherwise break current conservation. In this respect it is useful to consider that current (i), as current (c), yields contribution in both the kinematical regions (i) $p_{1}^{+}=k_{1}^{\prime+}-Q^{+}>0$ and (ii) $0>k_{1}^{\prime+}-Q^{+}=p_{1}^{+}$, as easily obtained from the kinematical constraint $p_{1}^{\prime+}=$ $k_{1}^{+}+Q^{+}>k_{1}^{\prime+}$ leading to $k_{1}^{+}>k_{1}^{\prime+}-Q^{+}=p_{1}^{+} \gtreqless 0$.

The peculiarity of the instantaneous terms in the treatment of the fermionic case is also illustrated by the cancellation of logarithmic singularities of the iterated ladder and stretched box diagrams of the Yukawa model [10,29]. This cancellation yields the full covariant form of the box diagram [29].

\section{CONCLUSION}

In this paper we propose a conserved electromagnetic current operator that acts on the valence component of the three-dimensional LF wave function of a two-fermion system. In order to obtain the LF current, we have ex- 
ploited the quasipotential approach to the BS equation [8] and then we have projected the relevant quantities onto the light-front hyperplane. This approach has been already applied to the BS equation for both boson and fermions $[9,10]$ and to the construction of the conserved current operator for two-boson systems [16].

The starting point of the reduction scheme to the LF is the QP approach to the BS equation with a proper auxiliary four-dimensional Green's function without instantaneous terms. Such terms, that represent the peculiar feature of a fermionic system, are recovered both in the kernel of the LF equation for the valence wave function and in the LF two-body current operator. The integration of the minus component of the particle four momenta in the relevant operators, namely, the Green's function, the T matrix, and etc., allows one to accomplish the desired LF projection of both eigenequation and em four current. Furthermore, we have identified a nonunitary operator, the reverse LF-time projection operator, that acts on the LF valence wave function and allows one to reconstruct the fourdimensional BS amplitude for bound or scattering states. Such an operator is nonunitary since the valence component does not carry the full normalization of the wave function (for a discussion of the probability of the higher Fock components in the Wick-Cutkosky model see Ref. [17]). The explicit expression of the reverse LF-time operator in terms of the quasipotential is essential for obtaining a LF three-dimensional current that fulfills the WTI, at any order of the quasipotential expansion. In particular, the reverse LF-time operator applied to a fourdimensional current generates a three-dimensional LF current. Such an application trivially leads to the equality between the matrix elements of the four current, evaluated by using the full BS amplitudes, and the matrix elements, of the LF current evaluated by using the valence wave functions corresponding to the previous BS amplitudes. Then the current conservation follows. This result is essential for identifying the ingredients to be used for demonstrating the WTI for the truncated LF current. In particular, we have defined the left and right LF charge operators (that do not contain interaction), and we have found the formal expression for the LF WTI, where the LF Green's functions, for the initial and final states, appear. The last step in our analysis is of particular relevance. Indeed, a naive truncation of the QP expansion that defines the LF current does not lead to a conserved current, while retaining all the contributions up to a given order in the effective interaction allows one to construct a LF truncated current that satisfies the WTI. In such a truncated WTI, the truncated initial and final Green's functions appear, as well as the same LF charge operators obtained in the full case. In particular, the truncated effective current operator can be put in correspondence to a sum over intermediate states [11] up to some maximal number of particles exchanged at a given LF time that flows from the initial to the final valence states due to the photo-absorption process. The truncation of the expansion of the quasipotential implies that the observables derived from the three-dimensional conserved current operator are frame dependent, but with gauge invariance correctly implemented. The covariance under Lorentz transformations of the full LF current operator, $j^{\mu}\left(K_{f}, K_{i}\right)$, is not greatly relevant, given the equality in Eq. (57) between the four-dimensional matrix elements of the covariant current and the corresponding threedimensional ones. However, since the truncated theory is necessary for obtaining workable approximations, the covariance properties of both the full and the truncated LF current will be investigated elsewhere [27]. Here, we can anticipate that the covariance under the seven kinematical LF transformations is satisfied by the LF current operator (and also by the valence wave functions) in both cases, with a suitable introduction of new factors in the vertical bar operation. For the truncated theory, the violation of covariance under dynamical LF transformations produces effects on the matrix elements of the current, that can be reduced at any desired accuracy, by increasing the order $n$ of the truncated quasipotential, namely, approaching the full theory. This necessarily leads to consider intermediate Fock states with larger and larger number of particles in the evaluation of the effective interaction. Moreover, a quantitative study of the effect of covariance violations was already performed in the computation of the masses of bound states for the bosonic model [9,25]. It was concluded that the expansion in the Fock space is rapidly converging for a given covariant model (see also, [5,30,31]). In particular the splitting between magnetic states of spin-1 composite bosons decreases when the kernel of the LF bound state equation is evaluated taking into account higher order terms, like stretched boxes, in a bosonic model [25].

Our procedure for constructing a truncated LF current has been illustrated in an actual case: the Yukawa model with chargeless boson exchange in ladder approximation. We have evaluated the LF current operator at the lowest nontrivial order and have explicitly checked the WardTakahashi identity for such a model. The role of instantaneous terms has been clarified and their relevance in producing two-body contributions has been emphasized.

Let us finally comment on possible problems about singularities and regularizations that can occur in the truncated LF current. Restricting to a model without selfenergies and vertex corrections, but considering in the kernel the ladder and two-body irreducible cross-ladder terms, the Bethe-Salpeter equation is finite allowing a solution. However, the projection of this equation to the light-front is plagued by infinities (see, e.g., [10,23,2931]). Recently it was shown that the finite covariant box amplitude in the Yukawa model is fully recovered when all terms in the light-front projection beyond the iterated box (i.e., stretched box and instantaneous terms) are obtained 
and that all the singularities are canceled [29]. Thus in general one could argue that regularization problems occur in the integration loops for the truncated current at order $n>1$, since the presence in $\Delta_{0}$ of the global propagation, described by $\tilde{G}_{0}(K)$, destroys the balance between singularities that allows for a finite result when only $G_{0}(K)$ is considered (see, e.g., [29]). For instance, an easy form of regularization can be introduced in $\tilde{G}_{0}(K)$ through a cutoff function, e.g., $\theta\left(\mu^{2}-M_{0}^{2}\right)$. Since the starting fourdimensional covariant BS model is finite, one expects that the effect of the scale $\mu$ vanishes if the QP expansion is not truncated.

However, the issue of renormalization of the nonperturbative bound state problem in the truncated QP expansion is subtle [24], and therefore the dependence upon the scale $\mu$ in the three-dimensional truncated theory should be carefully analyzed. This nontrivial elaboration on the renormalization issue has to be postponed to a future work.

In summary, we have proposed a systematic expansion of a conserved electromagnetic current operator within LF dynamics for two-fermion interacting systems, using the quasipotential approach to the Bethe-Salpeter equation. As to the future perspectives, we plan to apply such an interacting current for the investigation of inclusive and exclusive electromagnetic processes, like hadron form factors and deeply virtual photon scattering, after properly generalizing the present approach to fermion-antifermion systems [27]. It should be pointed out that the identification of the matrix elements of any operator in the fourdimensional space with the matrix elements of the corresponding three-dimensional operator acting on the valence wave function is a general procedure.

\section{ACKNOWLEDGMENTS}

This work was partially supported by the Brazilian agencies CNPq and FAPESP and by Ministero della Ricerca Scientifica e Tecnologica. J. A. O.M. and T.F. acknowledge the hospitality of the Dipartimento di Fisica, Università di Roma "Tor Vergata" and of Istituto Nazionale di Fisica Nucleare, Sezione Tor Vergata and Sezione Roma I.

\section{APPENDIX A: USEFUL IDENTITIES}

We introduce the following identities that will be useful in exploring the relation between LF and four-dimensional covariant quantities. It is straightforward to get that

$$
\begin{aligned}
& (\not p-m)\left(\not p_{\text {on }}+m\right)=\left(p^{2}-m^{2}\right) \frac{\gamma^{+}}{2 p^{+}}\left(\not p_{\text {on }}+m\right), \\
& (\not \text { on }+m)(\not p-m)=\left(p^{2}-m^{2}\right)\left(\not \text { on }_{\text {on }}+m\right) \frac{\gamma^{+}}{2 p^{+}} .
\end{aligned}
$$

These identities imply the following relations

$$
\begin{aligned}
& \left\langle k_{1}^{\prime-}\left|G_{0}^{-1}(K) \bar{G}_{0}(K)\right| k_{1}^{-}\right\rangle \\
& =\frac{\gamma_{1}^{+}}{2 k_{1}^{+}} \frac{\gamma_{2}^{+}}{2 k_{2}^{+}}\left(k_{1 \mathrm{on}}+m_{1}\right)\left(k_{2 \mathrm{on}}+m_{2}\right) \delta\left(k_{1}^{\prime-}-k_{1}^{-}\right), \\
& \left\langle k_{1}^{\prime-}\left|\bar{G}_{0}(K) G_{0}^{-1}(K)\right| k_{1}^{-}\right\rangle \\
& =\left(k_{1 \mathrm{on}}+m_{1}\right)\left(k_{2 \mathrm{on}}+m_{2}\right) \frac{\gamma_{1}^{+}}{2 k_{1}^{+}} \frac{\gamma_{2}^{+}}{2 k_{2}^{+}} \delta\left(k_{1}^{\prime-}-k_{1}^{-}\right) .
\end{aligned}
$$

Note the presence of the product $\gamma_{1}^{+} \gamma_{2}^{+}$that is essential for the definition of the LF charge operators, Eqs. (62) and (63). Exploiting $\left(\gamma^{+}\right)^{2}=0$, one obtains another useful identity

$$
(\not p+m) \frac{\gamma^{+}}{2 p^{+}}(\not p+m)=\left(\not \text { on }_{\text {on }}+m\right),
$$

with $p_{\text {on }}^{-}=\left(\vec{p}_{\perp}^{2}+m\right) / p^{+}$.

Using (i) Eqs. (A3) and (A5), and (ii) the explicit form of $\bar{G}_{0}(K)$, one gets

$$
g_{0}(K)=\left|\bar{G}_{0}(K) G_{0}^{-1}(K) \bar{G}_{0}(K)\right|,
$$

for the free three-dimensional propagator.

Now we can relate the interacting LF Green's function directly with the four-dimensional Green's function by evaluating

$$
\begin{aligned}
& \left|\bar{G}_{0}(K) G_{0}^{-1}(K) G(K) G_{0}^{-1}(K) \bar{G}_{0}(K)\right| \\
& \quad=\left|\bar{G}_{0}(K) G_{0}^{-1}(K) \bar{G}_{0}(K)\right|+\left|\bar{G}_{0}(K) T(K) \bar{G}_{0}(K)\right| \\
& \quad=g_{0}(K)+g_{0}(K) \bar{\Pi}_{0}(K) T(K) \Pi_{0}(K) g_{0}(K) \\
& \quad=g_{0}(K)+g_{0}(K) t(K) g_{0}(K)=g(K),
\end{aligned}
$$

where Eq. (34) for the definition of $t(K)$ was used.

\section{APPENDIX B: THE INTERACTING REVERSE LF PROJECTION OPERATOR}

In this appendix, we will prove some useful identities involving the interacting reverse LF projection operator.

The following relation between $\Pi(K)$ [see, the definition in Eq. (42)] and $T(K)$ can be obtained from Eqs. (27), (29), (31), (34), and (39). Indeed one has 


$$
\begin{aligned}
\Pi(K) & =\Pi_{0}(K)+\Delta_{0}(K) W(K) \Pi_{0}(K)=\Pi_{0}(K)+G_{0}(K) W(K) \Pi_{0}(K)-\Pi_{0}(K) g_{0}(K) \bar{\Pi}_{0}(K) W(K) \Pi_{0}(K) \\
& =\Pi_{0}(K)+G_{0}(K) W(K) \Pi_{0}(K)-\Pi_{0}(K) g_{0}(K) w(K)=\Pi_{0}(K) g_{0}(K) g^{-1}(K)+G_{0}(K) W(K) \Pi_{0}(K) g(K) g^{-1}(K) \\
& =\left[\Pi_{0}(K)+G_{0}(K) W(K) \Pi_{0}(K)+G_{0}(K) W(K) \Pi_{0}(K) g_{0}(K) t(K)\right] g_{0}(K) g^{-1}(K)= \\
& =\left[1+G_{0}(K) W(K)+G_{0}(K) W(K) \tilde{G}_{0}(K) T(K)\right] \Pi_{0}(K) g_{0}(K) g^{-1}(K)=\left[1+G_{0}(K) T(K)\right] \Pi_{0}(K) g_{0}(K) g^{-1}(K) .
\end{aligned}
$$

Furthermore, by using Eqs. (2) and (19), one gets

$$
\Pi(K)=G(K) G_{0}^{-1}(K) \bar{G}_{0}(K) \mid g^{-1}(K)
$$

that can be recast in the following form

$$
G^{-1}(K) \Pi(K)=G_{0}^{-1}(K) \bar{G}_{0}(K) \mid g^{-1}(K) .
$$

The analogous expression for $\bar{\Pi}(K)$ reads

$$
\bar{\Pi}(K) G^{-1}(K)=g^{-1}(K) \mid \bar{G}_{0}(K) G_{0}^{-1}(K) .
$$

\section{APPENDIX C: ZERO-ORDER LF CURRENT OPERATOR AND WTI}

In this appendix we will evaluate the matrix elements of the free LF current operator, Eq. (69), between free particle states and we will prove explicitly the WTI. The matrix elements of the free four-dimensional current operator are

$$
\begin{aligned}
\left\langle k_{1}\left|\mathcal{J}_{0}^{\mu}(Q)\right| p_{1}\right\rangle= & -2 \pi\left[e_{1} \gamma_{1}^{\mu} \delta^{4}\left(k_{1}-p_{1}-Q\right)\right. \\
& \left.\times\left(\left(\not \mathbb{K}_{f}-\not k_{1}\right)-m_{2}\right)\right] \\
& +\left[1 \rightarrow 2, k_{1} \rightarrow K_{f}-k_{1}, p_{1} \rightarrow K_{i}-p_{1}\right]
\end{aligned}
$$

where $Q^{\mu}=K_{f}^{\mu}-K_{i}^{\mu}$. The factor $(-2 \pi)$ is introduced in the current operator to make it compatible with the free Green's function, see Eq. (3).

Since $g_{0}^{-1}$ is the identity in the two-particle space, modulo some factors, to simplify the presentation let us consider in what follows only the relevant part of $j^{c \mu(0)}$ containing the $k^{-}$integration. Moreover, in order to make more fast the discussion related to the position of the poles, we will take profit of the $\theta$ functions present in the dropped $g_{0}^{-1}$ factors, that remind us we are dealing with particles in the external legs. Therefore, one has

$$
\begin{aligned}
& \Theta\left\langle k_{1}^{\prime+} \vec{k}_{1 \perp}^{\prime}\left\|\bar{G}_{0} \mathcal{J}_{0}^{\mu} \bar{G}_{0}\right\| k_{1}^{+} \vec{k}_{1 \perp}\right\rangle \\
& =-\frac{1}{(2 \pi)} \Theta \int d k_{1}^{-} \frac{e_{1} \delta\left(k_{1}^{\prime+}-k_{1}^{+}-Q^{+}\right) \delta^{2}\left(\vec{k}_{1 \perp}^{\prime}-\vec{k}_{1 \perp}-\vec{Q}_{\perp}\right)}{k_{1}^{\prime+} k_{1}^{+}\left(k_{1}^{-}+Q^{-}-k_{1 \text { on }}^{\prime-}+i \frac{\varepsilon}{k_{1}^{\prime+}}\right)\left(k_{1}^{-}-k_{1 \mathrm{on}}^{-}+i \frac{\varepsilon}{k_{1}^{+}}\right)} \frac{\left(k_{1 \mathrm{on}}^{\prime}+m_{1}\right) \gamma_{1}^{\mu}\left(k_{1 \mathrm{on}}+m_{1}\right)\left(\left(\not_{i}-\not k_{1}\right)_{\mathrm{on}}+m_{2}\right)}{\left(K_{i}^{+}-k_{1}^{+}\right)\left(K_{i}^{-}-k_{1}^{-}-\left(K_{i}-k_{1}\right)_{2 \mathrm{on}}^{-}+i \frac{\varepsilon}{K_{i}^{+}-k_{1}^{+}}\right)} \\
& \quad+1 \leftrightarrow 2,
\end{aligned}
$$

where $k_{1}^{\prime}=k_{1}+Q$, and $\Theta=\theta\left(k_{1}^{+}\right) \theta\left(k_{1}^{+\prime}\right) \theta\left(K_{i}^{+}-k_{1}^{+}\right) \theta\left(K_{f}^{+}-k_{1}^{+\prime}\right)$. It is important noting that, without profiting of the presence of $\Theta$ on the left of Eq. (C2), a lengthy discussion of the poles leads to the presence of $\Theta$ in the result. We have used the identities (A1) and (A5) to simplify the following combination that appears in the numerator of Eq. (C2)

$$
\left(k_{2 \mathrm{on}}+m_{2}\right)\left(k_{2}-m_{2}\right)\left(k_{2 \mathrm{on}}+m_{2}\right)=\left(k_{2}^{2}-m_{2}^{2}\right)\left(k_{2 \mathrm{on}}+m_{2}\right),
$$

with $k_{2}=K_{i}-k_{1}=K_{f}-k_{1}^{\prime}$.

Integrating over $k_{1}^{-}$and assuming that $K_{i}^{+}>0$ and $Q^{+} \geq 0$, without loss of generality, one gets that

$$
\begin{aligned}
\Theta\left\langle k_{1}^{\prime+} \vec{k}_{1 \perp}^{\prime}\left\|\bar{G}_{0} \mathcal{J}_{0}^{\mu} \bar{G}_{0}\right\| k_{1}^{+} \vec{k}_{1 \perp}\right\rangle= & i \Theta e_{1} \delta\left(k_{1}^{\prime+}-k_{1}^{+}-Q^{+}\right) \delta^{2}\left(\vec{k}_{1 \perp}^{\prime}-\vec{k}_{1 \perp}-\vec{Q}_{\perp}\right) \\
& \times \frac{\left(k_{1 \text { on }}^{\prime}+m_{1}\right) \gamma_{1}^{\mu}\left(k_{1 \text { on }}+m_{1}\right)\left(\left(\not_{i}-\not k_{1}\right)_{\text {on }}+m_{2}\right)}{k_{1}^{\prime+}\left(K_{i}^{+}-k_{1}^{+}\right) k_{1}^{+} \Delta_{f}^{(0)-} \Delta_{i}^{(0)-}}+1 \leftrightarrow 2
\end{aligned}
$$

where we have introduced the four-vector quantities $\Delta_{f}^{(0)}=K_{f}-k_{1 \text { on }}^{\prime}-\left(K_{f}-k_{1}^{\prime}\right)_{\text {on }}+i \epsilon \quad$ and $\quad \Delta_{i}^{(0)}=$ $K_{i}-k_{\text {lon }}-\left(K_{i}-k_{1}\right)_{\text {on }}+i \epsilon$ for convenience. Note that the minus component of $\Delta_{f}$ and $\Delta_{i}$ are the only nonvanishing ones. The on-minus-shell values of the individual momenta are

$$
\begin{aligned}
& k_{\text {1on }}^{\prime-}=\frac{\vec{k}_{1 \perp}^{\prime 2}+m_{1}^{2}}{k_{1}^{\prime+}} \\
& k_{\text {1on }}^{-}=\frac{\vec{k}_{1 \perp}^{2}+m_{1}^{2}}{k_{1}^{+}}
\end{aligned}
$$




$$
\begin{aligned}
& \left(K_{f}-k_{1}^{\prime}\right)_{\text {on }}^{-}=\frac{\left(\vec{K}_{f \perp}-\vec{k}_{1 \perp}^{\prime}\right)^{2}+m_{2}^{2}}{K_{f}^{+}-k_{1}^{\prime+}} \\
& \left(K_{i}-k_{1}\right)_{\text {on }}^{-}=\frac{\left(\vec{K}_{i \perp}-\vec{k}_{1 \perp}\right)^{2}+m_{2}^{2}}{K_{i}^{+}-k_{1}^{+}} .
\end{aligned}
$$

Taking into account the definition of $g_{0}(K)$, Eq. (16), and the matrix elements of $\hat{e}_{1 \mathrm{LF}}$, Eq. (65), we have

$$
\left\langle k_{1}^{\prime+} \vec{k}_{1 \perp}^{\prime}\left\|\bar{G}_{0} \mathcal{J}_{0}^{\mu} \bar{G}_{0}\right\| k_{1}^{+} \vec{k}_{1 \perp}\right\rangle=-i \frac{K_{i}^{+}-k_{1}^{+}}{2 m_{2}}\left\langle k_{1}^{\prime+}, \vec{k}_{1 \perp}^{\prime}\left|g_{0}\left(K_{f}\right) \gamma_{1}^{\mu} \hat{e}_{1, \mathrm{LF}} g_{0}\left(K_{i}\right)\right| k_{1}^{+} \vec{k}_{1 \perp}\right\rangle+1 \leftrightarrow 2 .
$$

Finally, by multiplying by the proper $g_{0}(K)^{-1}$, the matrix element of the free current operator can be written as

$$
\left\langle k_{1}^{\prime+} \vec{k}_{1 \perp}^{\prime}\left|j^{c(0) \mu}\right| k_{1}^{+} \vec{k}_{1 \perp}\right\rangle=-i \Theta \Lambda_{+}\left(k_{1 \mathrm{on}}^{\prime}\right) \gamma_{1}^{\mu} \Lambda_{+}\left(k_{1 \mathrm{on}}\right) \Lambda_{+}\left(\left(K_{i}-k_{1}\right)_{\mathrm{on}}\right) \frac{K_{i}^{+}-k_{1}^{+}}{2 m_{2}}\left\langle k_{1}^{\prime+}, \vec{k}_{1 \perp}^{\prime}\left|\hat{e}_{1, \mathrm{LF}}\right| k_{1}^{+} \vec{k}_{1 \perp}\right\rangle+1 \leftrightarrow 2 .
$$

To derive the WTI for the free LF current operator, we evaluate the four-divergence of the current by contracting it with $Q^{\mu}$. Since $\left(K_{f}-k_{1}^{\prime}\right)_{\text {on }}^{-}=\left(K_{i}-k_{1}\right)_{\text {on }}^{-}$, which follows from kinematical momentum conservation, the momentum transfer can be written in terms of $\Delta$ 's as, $Q=\Delta_{f}^{(0)}-\Delta_{i}^{(0)}+k_{1 \text { on }}^{\prime}-k_{\text {lon }}$, and thus

$$
\emptyset=\frac{\gamma^{+}}{2}\left(\Delta_{f}^{(0)-}-\Delta_{i}^{(0)-}\right)+k_{1 \mathrm{on}}^{\prime}-k_{\text {lon }}
$$

Then one has

$$
\Lambda_{+}\left(k_{\text {lon }}^{\prime}\right) \emptyset \Lambda_{+}\left(k_{1 \mathrm{on}}\right)=\Lambda_{+}\left(k_{1 \mathrm{on}}^{\prime}\right) \frac{\gamma_{1}^{+}}{2} \Lambda_{+}\left(k_{\mathrm{lon}}\right)\left(\Delta_{f}^{(0)-}-\Delta_{i}^{(0)-}\right) .
$$

From the above results, the four divergence of the free current becomes

$$
\begin{aligned}
\left\langle k_{1}^{\prime+} \vec{k}_{1 \perp}^{\prime}\left|Q \cdot j^{c(0)}\right| k_{1}^{+} \vec{k}_{1 \perp}\right\rangle= & -i \Theta \Lambda_{+}\left(k_{1 \mathrm{on}}^{\prime}\right) \frac{\gamma_{1}^{+}}{2} \Lambda_{+}\left(k_{1 \mathrm{on}}\right) \Lambda_{+}\left(\left(K_{i}-k_{1}\right)_{\mathrm{on}}\right) \frac{K_{i}^{+}-k_{1}^{+}}{2 m_{2}}\left(\Delta_{f}^{(0)-}-\Delta_{i}^{(0)-}\right)\left\langle k_{1}^{\prime+}, \vec{k}_{1 \perp}^{\prime}\left|\hat{e}_{1, \mathrm{LF}}\right| k_{1}^{+} \vec{k}_{1 \perp}\right\rangle \\
& +1 \leftrightarrow 2 . \\
= & \left\langle k_{1}^{\prime+} \vec{k}_{1 \perp}^{\prime}\left|\left[g_{0}\left(K_{f}\right)\right]^{-1} \hat{\mathcal{Q}}_{\mathrm{LF}}^{L}-\hat{\mathcal{Q}}_{\mathrm{LF}}^{R}\left[g_{0}\left(K_{i}\right)\right]^{-1}\right| k_{1}^{+} \vec{k}_{1 \perp}\right\rangle
\end{aligned}
$$

since the free resolvent that appears in Eq. (C13) comes from the following relations

$$
\begin{aligned}
& \Delta_{i}^{(0)-} \Lambda_{+}\left(k_{1 \mathrm{on}}\right) \Lambda_{+}\left(k_{2 \mathrm{on}}\right)=i \frac{2 m_{1}}{k_{1}^{+}} \frac{2 m_{2}}{K_{i}^{+}-k_{1}^{+}}\left[g_{0}\left(K_{i}\right)\right]^{-1} \\
& \Delta_{f}^{(0)-} \Lambda_{+}\left(k_{1 \mathrm{on}}^{\prime}\right) \Lambda_{+}\left(k_{2 \mathrm{on}}\right)=i \frac{2 m_{1}}{k_{1}^{\prime+}} \frac{2 m_{2}}{K_{f}^{+}-k_{1}^{\prime+}}\left[g_{0}\left(K_{f}\right)\right]^{-1} .
\end{aligned}
$$

Equation (C13) gives, as expected, the matrix elements of (70), i.e., the WTI for $n=0$.

\section{APPENDIX D: WTI FOR THE FIRST-ORDER LF CURRENT OPERATOR}

In this appendix we show the WTI for LF current, obtained by truncating at the first order the effective interaction, see Sec. VI. Let us rewrite the LF first-order current operator

$$
j^{c(1) \mu}=\bar{\Pi}_{0}\left[\mathcal{J}_{0}^{\mu}+\mathcal{J}_{I}^{\mu}+V \Delta_{0} \mathcal{J}_{0}^{\mu}+\mathcal{J}_{0}^{\mu} \Delta_{0} V\right] \Pi_{0}
$$

Using the current conservation for $\mathcal{J}_{0}^{\mu}$ and $\mathcal{J}_{I}^{\mu}$, see Eqs. (11) and (12), the four divergence is given by

$$
\begin{aligned}
Q^{\mu} j_{\mu}^{c(1)}= & g_{0}{ }^{-1} \hat{\mathcal{Q}}_{\mathrm{LF}}^{L}-\hat{\mathcal{Q}}_{\mathrm{LF}}^{R} g_{0}{ }^{-1}+\bar{\Pi}_{0}[(\hat{e} V-V \hat{e}) \\
& \left.+V \Delta_{0} G_{0}^{-1} \hat{e}-\hat{e} G_{0}^{-1} \Delta_{0} V\right] \Pi_{0} .
\end{aligned}
$$

Note that the term $\left[-V \Delta_{0} \hat{e} G_{0}^{-1} \Pi_{0}+\bar{\Pi}_{0} G_{0}^{-1} \hat{e} \Delta_{0} V\right]$ is not present in the above equation, since it is vanishing due to the absence of a Dirac structure in the operator $\hat{e}$ and because of Eqs. (A3), (A4), and (32).

Furthermore, by using the definitions of (i) $\Delta_{0}$, Eq. (31), (ii) $\tilde{G}_{0}$, Eq. (27), and (iii) $\hat{\mathcal{Q}}_{\mathrm{LF}}^{L}$, Eq. (62), and $\hat{\mathcal{Q}}_{\mathrm{LF}}^{R}$, Eq. (63), one has 


$$
\begin{aligned}
Q^{\mu} j_{\mu}^{c(1)} & =g_{0}{ }^{-1} \hat{\mathcal{Q}}_{\mathrm{LF}}^{L}-\hat{\mathcal{Q}}_{\mathrm{LF}}^{R} g_{0}{ }^{-1}-\bar{\Pi}_{0}\left[V \tilde{G}_{0} G_{0}^{-1} \hat{e}-\hat{e} G_{0}^{-1} \tilde{G}_{0} V\right] \Pi_{0} \\
& =g_{0}{ }^{-1} \hat{\mathcal{Q}}_{\mathrm{LF}}^{L}-\hat{\mathcal{Q}}_{\mathrm{LF}}^{R} g_{0}{ }^{-1}-\bar{\Pi}_{0}\left[V \Pi_{0} g_{0} \bar{\Pi}_{0} G_{0}^{-1} \hat{e}-\hat{e} G_{0}^{-1} \Pi_{0} g_{0} \bar{\Pi}_{0} V\right] \Pi_{0} \\
& =g_{0}{ }^{-1} \hat{\mathcal{Q}}_{\mathrm{LF}}^{L}-\hat{\mathcal{Q}}_{\mathrm{LF}}^{R} g_{0}{ }^{-1}-\left[w^{(1)}\left|\bar{G}_{0} G_{0}^{-1} \hat{e} \Pi_{0}-\bar{\Pi}_{0} \hat{e} G_{0}^{-1} G_{0}\right| w^{(1)}\right] \\
& =\left(g_{0}{ }^{-1}-w^{(1)}\right) \hat{\mathcal{Q}}_{\mathrm{LF}}^{L}-\hat{\mathcal{Q}}_{\mathrm{LF}}^{R}\left(g_{0}{ }^{-1}-w^{(1)}\right)=g_{1}{ }^{-1} \hat{\mathcal{Q}}_{\mathrm{LF}}^{L}-\hat{\mathcal{Q}}_{\mathrm{LF}}^{R} g_{1}{ }^{-1} .
\end{aligned}
$$

\section{APPENDIX E: WTI FOR ARBITRARY $n>1$ LF CURRENT OPERATOR}

The proof of WTI is based on the induction hypothesis. Once the current operator at order $n$ satisfies the WardTakahashi identity, we must demonstrate its validity for $n+1$. First note that

$$
\begin{aligned}
j^{c(n+1) \mu}= & j^{c(n) \mu}+\bar{\Pi}_{0}\left[W_{n} \Delta_{0} \mathcal{J}_{I}^{\mu}+\mathcal{J}_{I}^{\mu} \Delta_{0} W_{n}\right. \\
& +W_{n+1} \Delta_{0} \mathcal{J}_{0}^{\mu}+\mathcal{J}_{0}^{\mu} \Delta_{0} W_{n+1} \\
& +\sum_{i=1}^{n-1} W_{i} \Delta_{0} \mathcal{J}_{I}^{\mu} \Delta_{0} W_{n-i} \\
& \left.+\sum_{i=1}^{n} W_{i} \Delta_{0} \mathcal{J}_{0}^{\mu} \Delta_{0} W_{n-i+1}\right] \Pi_{0},
\end{aligned}
$$

where we have separated out the free term from the interacting one in the four-dimensional current using Eq. (5). Since $Q_{\mu} \mathcal{J}_{I}^{\mu}=\hat{e} V-V \hat{e}$, by induction, we have

$$
\begin{aligned}
Q^{\mu} j_{\mu}^{c(n+1)}= & g_{n}^{-1} \hat{\mathcal{Q}}_{\mathrm{LF}}^{L}-\hat{\mathcal{Q}}_{\mathrm{LF}}^{R} g_{n}^{-1}+\bar{\Pi}_{0}\left[W_{n} \Delta_{0}(\hat{e} V-V \hat{e})\right. \\
& +(\hat{e} V-V \hat{e}) \Delta_{0} W_{n} \\
& +W_{n+1} \Delta_{0}\left(G_{0}^{-1} \hat{e}-\hat{e} G_{0}^{-1}\right)+\left(G_{0}^{-1} \hat{e}-\hat{e} G_{0}^{-1}\right) \\
& \times \Delta_{0} W_{n+1}+\sum_{i=1}^{n-1} W_{i} \Delta_{0}(\hat{e} V-V \hat{e}) \Delta_{0} W_{n-i} \\
& \left.+\sum_{i=1}^{n} W_{i} \Delta_{0}\left(G_{0}^{-1} \hat{e}-\hat{e} G_{0}^{-1}\right) \Delta_{0} W_{n-i+1}\right] \Pi_{0} .
\end{aligned}
$$

In the above equation, the term $\bar{\Pi}_{0}\left[-W_{n+1} \Delta_{0} \hat{e} G_{0}^{-1}+\right.$ $\left.G_{0}^{-1} \hat{e} \Delta_{0} W_{n+1}\right] \Pi_{0}$ vanishes due to the absence of a Dirac structure in the operator $\hat{e}$ and because of Eqs. (A3), (A4), and (32). By using the same relations and the definition of $\tilde{G}_{0}$, Eq. (27), we can simplify the following term that appears in last sum, i.e.,

$$
\Delta_{0}\left(G_{0}^{-1} \hat{e}-\hat{e} G_{0}^{-1}\right) \Delta_{0}=\left(\hat{e} \Delta_{0}-\Delta_{0} \hat{e}\right) .
$$

Then, since by definition $V \Delta_{0} W_{i}=W_{i+1}$, we have

$$
\begin{aligned}
Q^{\mu} j_{\mu}^{c(n+1)}= & g_{n}^{-1} \hat{\mathcal{Q}}_{\mathrm{LF}}^{L}-\hat{\mathcal{Q}}_{\mathrm{LF}}^{R} g_{n}^{-1}+\bar{\Pi}_{0}\left[W_{n} \Delta_{0} \hat{e} V\right. \\
& -V \hat{e} \Delta_{0} W_{n}-W_{n+1} \tilde{G}_{0} G_{0}^{-1} \hat{e}+\hat{e} G_{0}^{-1} \tilde{G}_{0} W_{n+1} \\
& +\sum_{i=1}^{n-1} W_{i} \Delta_{0} \hat{e} W_{n-i+1}-\sum_{i=1}^{n-1} W_{i+1} \hat{e} \Delta_{0} W_{n-i} \\
& \left.+\sum_{i=1}^{n} W_{i}\left(\hat{e} \Delta_{0}-\Delta_{0} \hat{e}\right) W_{n-i+1}\right] \Pi_{0},
\end{aligned}
$$

and rearranging the terms we get

$$
\begin{aligned}
Q^{\mu} j_{\mu}^{c(n+1)}= & g_{n}^{-1} \hat{\mathcal{Q}}_{\mathrm{LF}}^{L}-\hat{\mathcal{Q}}_{\mathrm{LF}}^{R} g_{n}^{-1}+\bar{\Pi}_{0}\left[\hat{e} G_{0}^{-1} \tilde{G}_{0} W_{n+1}\right. \\
& -W_{n+1} \tilde{G}_{0} G_{0}^{-1} \hat{e}+\sum_{i=1}^{n} W_{i} \Delta_{0} \hat{e} W_{n-i+1} \\
& -\sum_{i=0}^{n-1} W_{i+1} \hat{e} \Delta_{0} W_{n-i} \\
& \left.+\sum_{i=1}^{n} W_{i}\left(\hat{e} \Delta_{0}-\Delta_{0} \hat{e}\right) W_{n-i+1}\right] \Pi_{0} .
\end{aligned}
$$

Since the sums cancel each other, we have

$$
\begin{aligned}
Q^{\mu} j_{\mu}^{c(n+1)}= & g_{n}^{-1} \hat{\mathcal{Q}}_{\mathrm{LF}}^{L}-\hat{\mathcal{Q}}_{\mathrm{LF}}^{R} g_{n}^{-1} \\
& +\bar{\Pi}_{0}\left[\hat{e} G_{0}^{-1} \tilde{G}_{0} W_{n+1}-W_{n+1} \tilde{G}_{0} G_{0}^{-1} \hat{e}\right] \\
& \times \Pi_{0}=g_{n}^{-1} \hat{\mathcal{Q}}_{\mathrm{LF}}^{L}-\hat{\mathcal{Q}}_{\mathrm{LF}}^{R} g_{n}^{-1} \\
& +\hat{\mathcal{Q}}_{\mathrm{LF}}^{R} w_{n+1}-w_{n+1} \hat{\mathcal{Q}}_{\mathrm{LF}}^{L}
\end{aligned}
$$

where the LF charge operators as given by Eqs. (62) and (63) have been introduced through

$$
\begin{aligned}
& \bar{\Pi}_{0} \hat{e} G_{0}^{-1} \tilde{G}_{0} W_{n+1} \Pi_{0}=\hat{Q}_{\mathrm{LF}}^{R} w_{n+1} \\
& \bar{\Pi}_{0} W_{n+1} \tilde{G}_{0} G_{0}^{-1} \hat{e} \Pi_{0}=w_{n+1} \hat{Q}_{\mathrm{LF}}^{L} .
\end{aligned}
$$

Finally, since $g_{n+1}^{-1}=g_{n}^{-1}-w_{n+1}$, one gets

$$
Q^{\mu} j_{\mu}^{c(n+1)}=g_{n+1}^{-1} \hat{Q}_{\mathrm{LF}}^{L}-\hat{Q}_{\mathrm{LF}}^{R} g_{n+1}^{-1} .
$$

Thus, by induction, we conclude that the LF electromagnetic current operator $j_{\mu}^{c(n)}$ is conserved at any given order $n$ of the quasipotential expansion, once the matrix elements are taken between eigenstates of $g_{n}^{-1}\left|\phi_{n}\right\rangle=0$. 


\section{APPENDIX F: INTERACTION-DEPENDENT PART OF THE LF FIRST-ORDER CURRENT OPERATOR IN LADDER APPROXIMATION}

The contribution of the interaction to the LF current operator in lowest order in ladder approximation comes from two-body irreducible amplitudes given by the second term in the rhs of Eq. (81). Using Eq. (31) for $\Delta_{0}$ one has

$$
\begin{aligned}
j^{c(1) \mu}-j^{c(0) \mu}= & \bar{\Pi}_{0} V G_{0} \mathcal{J}_{0}^{\mu} \Pi_{0}-w^{(1)} g_{0} j^{c(0) \mu} \\
& +\bar{\Pi}_{0} \mathcal{J}_{0}^{\mu} G_{0} V \Pi_{0}-j^{c(0) \mu} g_{0} w^{(1)},
\end{aligned}
$$

where $w^{(1)}=\bar{\Pi}_{0} V \Pi_{0}$ is the three-dimensional effective interaction [see Eq. (83)].

In the following we analyze the matrix elements of the first-order current operator relevant for the em processes in the spacelike region.

\section{Evaluation of the term $\left|\bar{G}_{\mathbf{0}} \mathcal{J}_{\mathbf{0}}^{\mu}(Q) G_{0} V \bar{G}_{\mathbf{0}}\right|$}

Let us start with the third term in the rhs. of Eq. (F1), $g_{0}^{-1}\left|\bar{G}_{0} \mathcal{J}_{0}^{\mu}(Q) G_{0} V \bar{G}_{0}\right| g_{0}^{-1}$, which is diagrammatically illustrated in Fig. 2. It is important to observe that this term contains the relevant pair production contribution, for $Q^{+}>0$ (cf. diagram (b) in Fig. 2). For the sake of simplicity, we can first drop the multiplicative factors $g_{0}^{-1}$ on the left and on the right, and we will consider the current only for particle 1 . However, we can take advantage of the $\theta$ functions contained in both $g_{0}^{-1}\left(K_{f}\right)$ and $g_{0}^{-1}\left(K_{i}\right)$, that greatly help in the discussion of the analytical integrations over $k_{1}^{-}$and $k_{1}^{\prime-}$. Therefore, we have to evaluate the following matrix element between free particle states

$$
\begin{aligned}
\Theta\left\langle k_{1}^{\prime+} \vec{k}_{1 \perp}^{\prime}\left\|\bar{G}_{0} \mathcal{J}_{0}^{\mu}(1) G_{0} V \bar{G}_{0}\right\| k_{1}^{+} \vec{k}_{1 \perp}\right\rangle= & i e_{1}\left(\frac{i g}{2 \pi}\right)^{2} \Theta \int d k_{1}^{\prime-} d k_{1}^{-} \frac{1}{k_{1}^{\prime+}\left(K_{f}^{+}-k_{1}^{\prime+}\right)} \frac{\not k_{1 \mathrm{on}}^{\prime \prime}+m_{1}}{\left(k_{1}^{\prime-}-k_{1 \mathrm{on}}^{\prime-}+i \frac{\varepsilon}{k_{1}^{\prime+}}\right)} \\
& \times \frac{\left(\not X_{f}-\not k_{1}^{\prime}\right)_{\mathrm{on}}+m_{2}}{\left(K_{f}^{-}-k_{1}^{\prime-}-\left(K_{f}-k_{1}^{\prime}\right)_{\mathrm{on}}^{-}+i \frac{\varepsilon}{\left.K_{f}^{+}-k_{1}^{\prime+}\right)}\right.} \gamma_{1}^{\mu} \frac{\not p_{1}+m_{1}}{p_{1}^{+}\left(p_{1}^{-}-p_{1 \mathrm{on}}^{-}+i \frac{\varepsilon}{p_{1}^{+}}\right)} \\
& \times \frac{\Gamma_{1}^{\alpha} \Gamma_{2 \alpha}}{\left(k_{1}^{+}-p_{1}^{+}\right)\left(k_{1}^{-}-p_{1}^{-}-\left(k_{1}-p_{1}\right)_{\mathrm{on}}^{-}+i \frac{\varepsilon}{k_{1}^{+}-p_{1}^{+}}\right)} \frac{1}{k_{1}^{+}\left(K_{i}^{+}-k_{1}^{+}\right)} \\
& \times \frac{k_{\text {lon }}+m_{1}}{\left(k_{1}^{-}-k_{1 \mathrm{on}}^{-}+i \frac{\varepsilon}{k_{1}^{+}}\right)} \frac{\left(\not K_{i}-\not k_{1}\right)_{\mathrm{on}}+m_{2}}{\left(K_{i}^{-}-k_{1}^{-}-\left(K_{i}-k_{1}\right)_{\mathrm{on}}^{-}+i \frac{\varepsilon}{K_{i}^{+}-k_{1}^{+}}\right)},
\end{aligned}
$$

where $\Theta=\theta\left(k_{1}^{+}\right) \theta\left(k_{1}^{+\prime}\right) \theta\left(K_{i}^{+}-k_{1}^{+}\right) \theta\left(K_{f}^{+}-k_{1}^{+\prime}\right) \quad$ and $p_{1}^{\mu}=k_{1}^{\prime \mu}-Q^{\mu}$. Equation (F2) is represented by the Feynman diagram shown in Fig. 1. The six poles in Eq. (F2) are

$$
\begin{aligned}
& k_{1 A}^{-}=k_{1 \mathrm{on}}^{-}-i \frac{\varepsilon}{k_{1}^{+}} \\
& k_{1 B}^{-}=K_{i}^{-}-\left(K_{i}-k_{1}\right)_{\mathrm{on}}^{-}+i \frac{\varepsilon}{K_{i}^{+}-k_{1}^{+}} \\
& k_{1 C}^{-}=p_{1}^{-}+\left(k_{1}-p_{1}\right)_{\mathrm{on}}^{-}-i \frac{\varepsilon}{k_{1}^{+}-p_{1}^{+}} \\
& k_{1 A}^{\prime-}=k_{1 \mathrm{on}}^{\prime-}-i \frac{\varepsilon}{k_{1}^{\prime+}} \\
& k_{1 B}^{\prime-}=K_{f}^{-}-\left(K_{f}-k_{1}^{\prime}\right)_{\mathrm{on}}^{-}+i \frac{\varepsilon}{K_{f}^{+}-k_{1}^{\prime+}} \\
& p_{1}^{-}=k_{1 C}^{\prime-}-Q^{-}=p_{1 \mathrm{on}}^{-}-i \frac{\varepsilon}{p_{1}^{+}}
\end{aligned}
$$

with the on-minus-shell definition of the respective momenta given by

$$
\begin{aligned}
k_{\text {lon }}^{-} & =\frac{\vec{k}_{1 \perp}^{2}+m_{1}^{2}}{k_{1}^{+}} \\
\left(K_{i}-k_{1}\right)_{\mathrm{on}}^{-} & =\frac{\left(\vec{K}_{i \perp}-\vec{k}_{1 \perp}\right)^{2}+m_{2}^{2}}{K_{i}^{+}-k_{1}^{+}} \\
\left(k_{1}-p_{1}\right)_{\mathrm{on}}^{-} & =\frac{\left(\vec{k}_{1 \perp}-\vec{p}_{1 \perp}\right)^{2}+\mu^{2}}{k_{1}^{+}-p_{1}^{+}} \\
p_{\text {lon }}^{-} & =\frac{\vec{p}_{1 \perp}^{2}+m_{1}^{2}}{p_{1}^{+}} \\
k_{1 \mathrm{on}}^{\prime-} & =\frac{\vec{k}_{1 \perp}^{\prime 2}+m_{1}^{2}}{k_{1}^{\prime+}} \\
\left(K_{f}-k_{1}^{\prime}\right)_{\mathrm{on}}^{-} & =\frac{\left(\vec{K}_{f \perp}-\vec{k}_{1 \perp}^{\prime}\right)^{2}+m_{2}^{2}}{K_{f}^{+}-k_{1}^{\prime+}} .
\end{aligned}
$$

The integrations over $k_{1}^{-}$and $k_{1}^{\prime}$ in Eq. (F2) are performed analytically using Cauchy's theorem with the conditions $K_{i}^{+}>0$ and $Q^{+} \geq 0$. As a result, we get the six contributions that appear in Fig. 2. Note that diagrams (d) and (e) are two-body reducible terms which are canceled by $j^{c(0) \mu} g_{0} w^{(1)}$ in Eq. (F1). Let us now discuss in detail the 
diagrams of Fig. 2, devoting a specific subsection to the pair diagram (b).

a. Diagrams (a), (c), (d), (e), and $(f)$ for $p_{1}^{+}=$ $k_{1}^{\prime+}-Q^{+} \geq 0$

Let us start considering the kinematical region $p_{1}^{+}=$ $k_{1}^{\prime+}-Q^{+} \geq 0$ and $k_{1}^{+}>k_{1}^{\prime+}-Q^{+}$. We first perform the analytical integration of Eq. (F2) on $k_{1}^{-}$. The poles $k_{1 A}^{-}$and $k_{1 C}^{-}$belong to the lower semiplane, while $k_{1 B}^{-}$lays in the upper semiplane. The result obtained integrating in the upper semiplane contains contributions corresponding to diagrams (a), (c) and (d), viz.,

$$
\begin{aligned}
\Theta\left\langle k_{1}^{\prime+} \vec{k}_{1 \perp}^{\prime}\left\|\bar{G}_{0} \mathcal{J}_{0}^{\mu}(1) G_{0} V \bar{G}_{0}\right\| k_{1}^{+} \vec{k}_{1 \perp}\right\rangle_{(a)+(c)+(d)=} & e_{1} \frac{(i g)^{2}}{2 \pi} \Theta \theta\left(p_{1}^{+}\right) \theta\left(k_{1}^{+}-p_{1}^{+}\right) \int d k_{1}^{\prime-} \frac{1}{k_{1}^{\prime+}\left(K_{f}^{+}-k_{1}^{\prime+}\right)} \frac{\not k_{1 \mathrm{on}}^{\prime \prime}+m_{1}}{\left(k_{1}^{\prime-}-k_{1 \mathrm{on}}^{-}+i \frac{\varepsilon}{k_{1}^{\prime+}}\right)} \\
& \times \frac{\left(\not X_{f}-\not k_{1}^{\prime}\right)_{\mathrm{on}}+m_{2}}{\left[K_{f}^{-}-k_{1}^{\prime-}-\left(K_{f}-k_{1}^{\prime}\right)_{\mathrm{on}}^{-}+i \frac{\varepsilon}{K_{f}^{+}-k_{1}^{\prime+}}\right]} \frac{1}{\left(k_{1}^{+}-p_{1}^{+}\right) p_{1}^{+}} \\
& \times \frac{\gamma_{1}^{\mu}}{\left[K_{f}^{-}-k_{1}^{\prime-}-\left(K_{i}-k_{1}\right)_{\mathrm{on}}^{-}+i \frac{\varepsilon}{K_{i}^{+}-k_{1}^{+}}-\left(k_{1}-p_{1}\right)_{\mathrm{on}}^{-}+i \frac{\varepsilon}{\left.k_{1}^{+}-p_{1}^{+}\right]}\right.} \\
& \times\left[\frac{\not p_{\mathrm{on}}+m_{1}}{\left(k_{1}^{\prime-}-Q^{-}-p_{1 \mathrm{on}}^{-}+i \frac{\varepsilon}{p_{1}^{+}}\right)}+\frac{\gamma_{1}^{+}}{2}\right] \frac{\Gamma_{1}^{\alpha} \Gamma_{2 \alpha}}{k_{1}^{+}\left(K_{i}^{+}-k_{1}^{+}\right)} \\
& \times \frac{\left(\not k_{1 \mathrm{on}}+m_{1}\right)\left[\left(\not K_{i}-\not k_{1}\right)_{\mathrm{on}}+m_{2}\right]}{\left[K_{i}^{-}-\left(K_{i}-k_{1}\right)_{\mathrm{on}}^{-}+i \frac{\varepsilon}{K_{i}^{+}-k_{1}^{+}}-k_{1 \mathrm{on}}^{-}+\frac{i \varepsilon}{k_{1}^{+}}\right]}
\end{aligned}
$$

Note that (i) the instantaneous term, proportional to $\gamma_{1}^{+}$, leading to a first contribution to the current (c) shown in Fig. 2 , is explicitly separated out in Eq. (F5), (ii) the global propagation of the initial state, i.e., $1 /\left[K_{i}^{-}-\left(K_{i}-k_{1}\right)_{\text {on }}^{-}-k_{\text {lon }}^{-}\right]$will be canceled by $g_{0}\left(K_{i}\right)^{-1}$.

In order to separate the processes corresponding to diagrams (a) and (d) of Fig. 2, we make use of the identity

$$
\begin{aligned}
\frac{1}{\left(K_{f}^{-}-k_{1}^{\prime-}-\left(K_{i}-k_{1}\right)_{\mathrm{on}}^{-}+i \frac{\varepsilon}{K_{i}^{+}-k_{1}^{+}}-\left(k_{1}-p_{1}\right)_{\mathrm{on}}^{-}+i \frac{\varepsilon}{\bar{k}_{1}^{+}-p_{1}^{+}}\right)} \frac{1}{\left(k_{1}^{\prime-}-Q^{-}-p_{1 \mathrm{on}}^{-}+i \frac{\varepsilon}{p_{1}^{+}}\right)} \\
=\left[\frac{1}{\left(K_{f}^{-}-k_{1}^{\prime-}-\left(K_{i}-k_{1}\right)_{\mathrm{on}}^{-}+i \frac{\varepsilon}{K_{i}^{+}-k_{1}^{+}}-\left(k_{1}-p_{1}\right)_{\mathrm{on}}^{-}+i \frac{\varepsilon}{k_{1}^{+}-p_{1}^{+}}\right)}+\frac{1}{\left(k_{1}^{\prime-}-Q^{-}-p_{1 \mathrm{on}}^{-}+i \frac{\varepsilon}{p_{1}^{+}}\right)}\right] \\
\quad \times \frac{1}{K_{i}^{-}-p_{\text {lon }}^{-}+i \frac{\varepsilon}{p_{1}^{+}}-\left(K_{i}-k_{1}\right)_{\mathrm{on}}^{-}+i \frac{\varepsilon}{K_{i}^{+}-k_{1}^{+}}-\left(k_{1}-p_{1}\right)_{\mathrm{on}}^{-}+i \frac{\varepsilon}{k_{1}^{+}-p_{1}^{+}}},
\end{aligned}
$$

and integrate Eq. (F5) analytically on $k_{1}^{\prime-}$. The first term in the square brackets in Eq. (F6) generates the contribution illustrated by diagram (a) in Fig. 2, once we take the residue at the pole $k_{1 A}^{\prime}$, in the lower semiplane, and multiply on the left by $g_{0}\left(K_{f}\right)^{-1}$ and on the right by $g_{0}\left(K_{i}\right)^{-1}$ [cf. Eq. (17)]. The second term in the square brackets leads to the contribution of diagram (d), a two-body reducible term, which is canceled out by one of the contributions in $j^{c(0) \mu} g_{0} w^{(1)}$, see Eq. (F1). Diagram (a) is given by

$$
\begin{aligned}
\left\langle k_{1}^{\prime+} \vec{k}_{1 \perp}^{\prime}\left|j^{c(1) \mu}\right| k_{1}^{+} \vec{k}_{1 \perp}\right\rangle_{(a)}= & i e_{1}\left(2 m_{1}\right)(i g)^{2} \Theta \frac{\theta\left(k_{1}^{+}-p_{1}^{+}\right)}{\left(k_{1}^{+}-p_{1}^{+}\right) p_{1}^{+}} \frac{\theta\left(p_{1}^{+}\right)}{\Delta_{f}^{(1 a)-} \Delta_{i}^{(1 a)-}} \Lambda_{+}\left(k_{1 \mathrm{on}}^{\prime}\right) \gamma_{1}^{\mu} \Lambda_{+}\left(p_{1 \mathrm{on}}\right) \Gamma_{1}^{\alpha} \Lambda_{+}\left(k_{1 \mathrm{on}}\right) \\
& \times \Lambda_{+}\left(\left(K_{f}-k_{1}^{\prime}\right)_{\mathrm{on}}\right) \Gamma_{2^{\alpha}} \Lambda_{+}\left(\left(K_{i}-k_{1}\right)_{\mathrm{on}}\right) .
\end{aligned}
$$

The four-vectors $\Delta_{f}^{(1 a)}$ and $\Delta_{i}^{(1 a)}$ are the following combinations of the four momenta

$$
\Delta_{f}^{(1 a)}=K_{f}-k_{\text {lon }}^{\prime}-\left(K_{i}-k_{1}\right)_{\mathrm{on}}-\left(k_{1}-p_{1}\right)_{\text {on }}+i \varepsilon \quad \Delta_{i}^{(1 a)}=K_{i}-p_{\text {lon }}-\left(K_{i}-k_{1}\right)_{\mathrm{on}}-\left(k_{1}-p_{1}\right)_{\mathrm{on}}+i \varepsilon .
$$

The minus components yield the three-body (two fermions and the exchanged boson) global propagation in the final and initial states, respectively.

The instantaneous term (c), in the kinematical region under consideration in this subsection, can be obtained from the same pole $k_{1 A}^{\prime}$. Then one has 


$$
\begin{aligned}
\left\langle k_{1}^{\prime+} \vec{k}_{1 \perp}^{\prime}\left|j^{c(1) \mu}\right| k_{1}^{+} \vec{k}_{1 \perp}\right\rangle_{(c)}^{I}= & \frac{i}{2} e_{1}(i g)^{2} \Theta \frac{\theta\left(p_{1}^{+}\right)}{p_{1}^{+}} \frac{\theta\left(k_{1}^{+}-p_{1}^{+}\right)}{\left(k_{1}^{+}-p_{1}^{+}\right) \Delta_{f}^{(1 a)-}} \Lambda_{+}\left(k_{1 \mathrm{on}}^{\prime}\right) \gamma_{1}^{\mu} \gamma_{1}^{+} \Gamma_{1}^{\alpha} \Lambda_{+}\left(k_{\mathrm{lon}}\right) \Lambda_{+}\left(\left(K_{f}-k_{1}^{\prime}\right)_{\mathrm{on}}\right) \\
& \times \Gamma_{2 \alpha} \Lambda_{+}\left(\left(K_{i}-k_{1}\right)_{\mathrm{on}}\right) .
\end{aligned}
$$

Let us then consider the kinematical region where $p_{1}^{+}=k_{1}^{\prime+}-Q^{+}>k_{1}^{+}$. We first perform the analytical integration of Eq. (F2) in $k_{1}^{-}$by calculating the residue at the pole $k_{1 A}^{-}$in the lower semiplane, where only this pole appears. Then in this region we obtain two contributions from Eq. (F2): a reducible one (cf. diagram (e) in Fig. 2) and an irreducible one given by diagram (f), namely, an instantaneous term. Diagram (e) is canceled by the remaining part of $j^{c(0) \mu} g_{0} w^{(1)}$. The result corresponding to diagram (f) is

$$
\begin{aligned}
\Theta\left\langle k_{1}^{\prime+} \vec{k}_{1 \perp}^{\prime}\left\|\bar{G}_{0} \mathcal{J}_{0}^{\mu}(Q) G_{0} V \bar{G}_{0}\right\| k_{1}^{+} \vec{k}_{1 \perp}\right\rangle_{(f)}= & e_{1} \frac{(i g)^{2}}{2 \pi} \Theta \theta\left(p_{1}^{+}\right) \theta\left(p_{1}^{+}-k_{1}^{+}\right) \int d k_{1}^{\prime-} \frac{1}{k_{1}^{\prime+}\left(K_{f}^{+}-k_{1}^{\prime+}\right)} \frac{k_{1 \mathrm{on}}^{\prime \prime}+m_{1}}{\left(k_{1}^{\prime-}-k_{1 \mathrm{on}}^{\prime-}+i \frac{\varepsilon}{k_{1}^{(+}}\right)} \\
& \times \frac{\left(\not K_{f}-\not k_{1}^{\prime \prime}\right)_{\mathrm{on}}+m_{2}}{\left[K_{f}^{-}-k_{1}^{\prime-}-\left(K_{f}-k_{1}^{\prime}\right)_{\mathrm{on}}^{-}+i \frac{\varepsilon}{K_{f}^{+}-k_{1}^{\prime+}}\right]} \frac{1}{\left(p_{1}^{+}-k_{1}^{+}\right) p_{1}^{+}} \\
& \times \frac{\gamma_{1}^{\mu}}{\left[k_{1}^{\prime-}-Q^{-}-k_{1 \mathrm{on}}^{-}-\left(p_{1}-k_{1}\right)_{\mathrm{on}}^{-}+i \frac{\varepsilon}{k_{1}^{+}}+i \frac{\varepsilon}{p_{1}^{+}-k_{1}^{+}}\right]} \frac{\gamma_{1}^{+}}{2} \\
& \times \frac{\Gamma_{1}^{\alpha} \Gamma_{2 \alpha}}{k_{1}^{+}\left(K_{i}^{+}-k_{1}^{+}\right)} \frac{\left(k_{1 \mathrm{on}}+m_{1}\right)\left(\left(\not K_{i}-\not k_{1}\right)_{\mathrm{on}}+m_{2}\right)}{\left[K_{i}^{-}-\left(K_{i}-k_{1}\right)_{\mathrm{on}}^{-}+i \frac{\varepsilon}{K_{i}^{+}-k_{1}^{+}-k_{1 \mathrm{on}}^{-}+i \frac{\varepsilon}{k_{1}^{+}}} .\right.}
\end{aligned}
$$

The analytical integration of Eq. (F10) is easily performed by calculating the residue at the pole $k_{1 B}^{\prime-}$ in the upper semiplane. Therefore, the contribution from the instantaneous term for $k_{1}^{\prime+}-Q^{+}>k_{1}^{+}$, represented by diagram (f), is

$$
\begin{aligned}
\left\langle k_{1}^{\prime+} \vec{k}_{1 \perp}^{\prime}\left|j^{c(1) \mu}\right| k_{1}^{+} \vec{k}_{1 \perp}\right\rangle_{(f)}= & \frac{i}{2} e_{1}(i g)^{2} \Theta \frac{\theta\left(p_{1}^{+}\right)}{p_{1}^{+}} \frac{\theta\left(p_{1}^{+}-k_{1}^{+}\right)}{\left(p_{1}^{+}-k_{1}^{+}\right) \Delta_{i}^{(1 f)-}} \Lambda_{+}\left(k_{1 \mathrm{on}}^{\prime}\right) \gamma_{1}^{\mu} \gamma_{1}^{+} \Gamma_{1}^{\alpha} \Lambda_{+}\left(k_{1 \mathrm{on}}\right) \Lambda_{+}\left(\left(K_{f}-k_{1}^{\prime}\right)_{\mathrm{on}}\right) \\
& \times \Gamma_{2^{\alpha}} \Lambda_{+}\left(\left(K_{i}-k_{1}\right)_{\mathrm{on}}\right)
\end{aligned}
$$

where in the denominator appears the minus component of the following four momentum

$$
\Delta_{i}^{(1 f)}=K_{i}-k_{\text {on }}-\left(K_{f}-k_{1}^{\prime}\right)_{\mathrm{on}}-\left(p_{1}-k_{1}\right)_{\mathrm{on}}
$$

which yields the intermediate propagation of the three-body system composed by two fermions and a boson.

$$
\text { b. Diagrams (b) and (c) for } 0 \geq k_{1}^{\prime+}-Q^{+}=p_{1}^{+}
$$

In the kinematical region where $0 \geq k_{1}^{\prime+}-Q^{+}=p_{1}^{+}$, the nonvanishing result can be obtained from the pole $k_{1 B}^{-}$in the upper semiplane, as in Eq. (F5). Then one has to consider the pole $k_{1 A}^{\prime-}$, when the integration over $k_{1}^{\prime-}$ is performed. Note that in Eq. (F5) $\theta\left(p_{1}^{+}\right)$has now to be substituted by $\theta\left(-p_{1}^{+}\right)$. The resulting matrix element of the current operator (b) is

$$
\begin{aligned}
\left\langle k_{1}^{\prime+} \vec{k}_{1 \perp}^{\prime}\left|j^{c(1) \mu}\right| k_{1}^{+} \vec{k}_{1 \perp}\right\rangle_{(b)}= & -i e_{1}\left(2 m_{1}\right)(i g)^{2} \Theta \frac{\theta\left(-p_{1}^{+}\right)}{p_{1}^{+}} \frac{\theta\left(k_{1}^{+}-p_{1}^{+}\right)}{\left(k_{1}^{+}-p_{1}^{+}\right) \Delta_{f}^{(1 a)-} \Delta_{\gamma}^{(1 b)-}} \Lambda_{+}\left(k_{1 \mathrm{on}}^{\prime}\right) \gamma_{1}^{\mu} \Lambda_{+}\left(p_{1 \mathrm{on}}\right) \Gamma_{1}^{\alpha} \Lambda_{+}\left(k_{1 \mathrm{on}}\right) \\
& \times \Lambda_{+}\left(\left(K_{f}-k_{1}^{\prime}\right)_{\mathrm{on}}\right) \Gamma_{2 \alpha} \Lambda_{+}\left(\left(K_{i}-k_{1}\right)_{\mathrm{on}}\right),
\end{aligned}
$$

where the combination $\Delta_{\gamma}^{(1 b)}$ of four momenta is

$$
\Delta_{\gamma}^{(1 b)}=Q-k_{\text {lon }}^{\prime}+p_{\text {1on }}+i \varepsilon .
$$

Finally one has to compute the two-body current due to the instantaneous term (c), which in this kinematical region is given by 


$$
\begin{aligned}
\left\langle k_{1}^{\prime+} \vec{k}_{1 \perp}^{\prime}\left|j^{c(1)_{\mu}}\right| k_{1}^{+} \vec{k}_{1 \perp}\right\rangle_{(c)}^{I I}= & \frac{i}{2} e_{1}(i g)^{2} \Theta \frac{\theta\left(-p_{1}^{+}\right)}{p_{1}^{+}} \frac{\theta\left(k_{1}^{+}-p_{1}^{+}\right)}{\left(k_{1}^{+}-p_{1}^{+}\right) \Delta_{f}^{(1 a)-}} \Lambda_{+}\left(k_{1 \mathrm{on}}^{\prime}\right) \gamma_{1}^{\mu} \gamma_{1}^{+} \Gamma_{1}^{\alpha} \Lambda_{+}\left(k_{1 \mathrm{on}}\right) \Lambda_{+}\left(\left(K_{f}-k_{1}^{\prime}\right)_{\mathrm{on}}\right) \\
& \left.\times \Gamma_{2 \alpha} \Lambda_{+}\left(K_{i}-k_{1}\right)_{\mathrm{on}}\right) .
\end{aligned}
$$

\section{Evaluation of the term $\left|\bar{G}_{\mathbf{0}} V G_{\mathbf{0}} \mathcal{J}_{\mathbf{0}}^{\mu}(Q) \bar{G}_{\mathbf{0}}\right|$}

Let us now discuss the first contribution in Eq. (F1), $g_{0}^{-1}\left|\bar{G}_{0} V G_{0} \mathcal{J}_{0}^{\mu}(Q) \bar{G}_{0}\right| g_{0}^{-1}$, depicted by diagrams (g) to (i) in Fig. 3. It is worth noting that the pair contribution is not present due to the conservation of the plus momentum component. For the sake of simplicity, the two reducible diagrams, which are present in this first term and are canceled by the term $w^{(1)} g_{0} j^{c(0) \mu}$ of Eq. (F1) in an analogous way as we have already discussed for diagrams (e) and (d) of Fig. 2, are not shown in Fig. 3. As we did for the third term in Eq. (F1), we evaluate first the matrix elements of the relevant part of the contribution under consideration, i.e.,

$$
\begin{aligned}
& \Theta\left\langle k_{1}^{\prime+} \vec{k}_{1 \perp}^{\prime}\left\|\bar{G}_{0} V G_{0} \mathcal{J}_{0}^{\mu}(1) \bar{G}_{0}\right\| k_{1}^{+} \vec{k}_{1 \perp}\right\rangle=i e_{1}\left(\frac{i g}{2 \pi}\right)^{2} \Theta \int d k_{1}^{\prime-} d k_{1}^{-} \frac{1}{k_{1}^{\prime+}\left(K_{f}^{+}-k_{1}^{\prime+}\right)} \frac{k_{1 \text { on }}^{\prime \prime}+m_{1}}{\left(k_{1}^{\prime-}-k_{1 \text { on }}^{\prime-}+i \frac{\varepsilon}{k_{1}^{\prime \prime}}\right)} \\
& \times \frac{\left(\not K_{f}-\not k_{1}^{\prime \prime}\right)_{\mathrm{on}}+m_{2}}{\left(K_{f}^{-}-k_{1}^{\prime-}-\left(K_{f}-k_{1}^{\prime}\right)_{\mathrm{on}}^{-}+i \frac{\varepsilon}{K_{f}^{+}-k_{1}^{\prime+}}\right)} \\
& \times \frac{\Gamma_{1}^{\alpha} \Gamma_{2 \alpha}}{\left(k_{1}^{\prime+}-p_{1}^{\prime+}\right)\left(k_{1}^{\prime-}-p_{1}^{--}-\left(k_{1}^{\prime}-p_{1}^{\prime}\right)_{\mathrm{on}}^{-}+i \frac{\varepsilon}{k_{1}^{\prime+}-p_{1}^{\prime+}}\right)} \\
& \times \frac{\not p_{1}^{\prime}+m_{1}}{p_{1}^{\prime+}\left(p_{1}^{\prime-}-p_{\text {lon }}^{\prime-}+i \frac{\varepsilon}{\left.p_{1}^{\prime+}\right)}\right.} \gamma_{1}^{\mu} \frac{1}{k_{1}^{+}\left(K_{i}^{+}-k_{1}^{+}\right)} \frac{k_{1 \text { on }}+m_{1}}{\left(k_{1}^{-}-k_{\text {lon }}^{-}+i \frac{\varepsilon}{k_{1}^{+}}\right)} \\
& \times \frac{\left(\not K_{i}-\not_{1}\right)_{\mathrm{on}}+m_{2}}{\left(K_{i}^{-}-k_{1}^{-}-\left(K_{i}-k_{1}\right)_{\mathrm{on}}^{-}+i \frac{\varepsilon}{K_{i}^{+}-k_{1}^{+}}\right)} \text {, }
\end{aligned}
$$

where the intermediate momentum is changed now to $p_{1}^{\prime \mu}=k_{1}^{\mu}+Q^{\mu}$. In order to analytically evaluate the integral, in addition to the poles $k_{1 A}^{-}, k_{1 B}^{-}, k_{1 A}^{\prime-}, k_{1 B}^{\prime-}$, given in Eq. (F3), we have to consider the following ones

$$
k_{1 D}^{-}+Q^{-}=p_{1 \mathrm{on}}^{\prime-}-i \frac{\varepsilon}{p_{1}^{\prime+}} \quad k_{1 D}^{\prime-}=p_{1}^{\prime-}+\left(k_{1}^{\prime}-p_{1}^{\prime}\right)_{\mathrm{on}}^{-}-i \frac{\varepsilon}{k_{1}^{\prime+}-p_{1}^{\prime+}}
$$

with the on-minus-shell definitions given by

$$
p_{1 \mathrm{on}}^{\prime-}=\frac{\vec{p}_{1 \perp}^{\prime 2}+m_{1}^{2}}{p_{1}^{\prime+}} \quad\left(k_{1}^{\prime}-p_{1}^{\prime}\right)_{\mathrm{on}}^{-}=\frac{\left(\vec{k}_{1 \perp}^{\prime}-\vec{p}_{1 \perp}^{\prime}\right)^{2}+\mu^{2}}{k_{1}^{\prime+}-p_{1}^{\prime+}}
$$

As already said in Sec. VII, we have always $p_{1}^{\prime+}>0$ and therefore no pair contribution can be generated from this term. In what follows we consider two regions: (i) $k_{1}^{\prime+}>p_{1}^{\prime+}=k_{1}^{+}+Q^{+}$and (ii) $p_{1}^{\prime+}=k_{1}^{+}+Q^{+}>k_{1}^{\prime+}$. Moreover the definition of $p_{1}^{\prime \mu}$ suggests to start with the integration over $k_{1}^{\prime-}$. In this way we have to discuss only three poles.

\section{a. Diagrams (g) and (h) for $k_{1}^{\prime+}>p_{1}^{\prime+} \geq 0$}

From the residue at the pole $k_{1 B}^{\prime-}$ one gets 


$$
\begin{aligned}
& \Theta\left\langle k_{1}^{\prime+} \vec{k}_{1 \perp}^{\prime}\left\|\bar{G}_{0} V G_{0} \mathcal{J}_{0}^{\mu}(1) \bar{G}_{0}\right\| k_{1}^{+} \vec{k}_{1 \perp}\right\rangle_{(g)+(h)} \\
& =e_{1} \frac{(i g)^{2}}{2 \pi} \Theta \theta\left(k_{1}^{\prime+}-p_{1}^{\prime+}\right) \int d k_{1}^{-} \frac{1}{k_{1}^{\prime+}\left(K_{f}^{+}-k_{1}^{\prime+}\right)} \frac{\not c}{\left[K_{f}^{-}-\left(K_{f}-k_{1}^{\prime}\right)_{\text {on }}^{-}-k_{1 \mathrm{on}}^{\prime-}+i \frac{\varepsilon}{k_{1}^{\prime+}}+i \frac{\varepsilon}{K_{f}^{+}-k_{1}^{\prime \prime}}\right]}\left[\left(\not K_{f}-\not k_{1}^{\prime \prime}\right)_{\mathrm{on}}+m_{2}\right] \\
& \times \frac{\Gamma_{1}^{\alpha} \Gamma_{2 \alpha}}{\left(k_{1}^{\prime+}-p_{1}^{\prime+}\right)\left[K_{f}^{-}-\left(K_{f}-k_{1}^{\prime}\right)_{\mathrm{on}}^{-}-p_{1}^{\prime-}-\left(k_{1}^{\prime}-p_{1}^{\prime}\right)_{\mathrm{on}}^{-}+i_{\frac{\varepsilon}{k_{1}^{\prime \prime}-p_{1}^{\prime+}}+i \frac{\varepsilon}{K_{f}^{+}-k_{1}^{\prime \prime}}}\right]}\left[\frac{\not p_{1 \mathrm{on}}^{\prime}+m_{1}}{p_{1}^{\prime+}\left(p_{1}^{\prime-}-p_{\text {on }}^{\prime-}+i \frac{\varepsilon}{\left.p_{1}^{\prime \prime}\right)}\right.}+\frac{\gamma^{+}}{2 p_{1}^{\prime+}}\right] \\
& \times \gamma_{1}^{\mu} \frac{1}{k_{1}^{+}\left(K_{i}^{+}-k_{1}^{+}\right)} \frac{k_{1 \mathrm{on}}+m_{1}}{\left(k_{1}^{-}-k_{\text {lon }}^{-}+i \frac{\varepsilon}{k_{1}^{+}}\right)} \frac{\left(\not K_{i}-\not K_{1}\right)_{\mathrm{on}}+m_{2}}{\left(K_{i}^{-}-k_{1}^{-}-\left(K_{i}-k_{1}\right)_{\mathrm{on}}^{-}+i \frac{\varepsilon}{K_{i}^{+}-k_{1}^{+}}\right)} .
\end{aligned}
$$

Adopting the same strategy as in Eq. (F6), one obtains (i) the contribution illustrated by diagram (g) in Fig. 3 and a reducible term (not shown in Fig. 3), (ii) the instantaneous term (h). In order to separate the irreducible contribution from the reducible one, we exploit the following identity

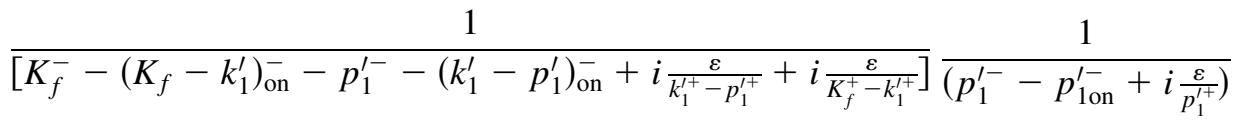

$$
\begin{aligned}
& =\left[\frac{1}{\left[K_{f}^{-}-\left(K_{f}-k_{1}^{\prime}\right)_{\mathrm{on}}^{-}-p_{1}^{--}-\left(k_{1}^{\prime}-p_{1}^{\prime}\right)_{\mathrm{on}}^{-}+i_{\frac{\varepsilon}{k_{1}^{\prime+}-p_{1}^{\prime+}}+i \frac{\varepsilon}{K_{f}^{+}-k_{1}^{\prime \prime}}}\right.}+\frac{1}{\left(p_{1}^{\prime-}-p_{\mathrm{on}}^{\prime-}+i \frac{\varepsilon}{\left.p_{1}^{\prime+}\right)}\right.}\right] \\
& \times \frac{1}{\left[K_{f}^{-}-\left(K_{f}-k_{1}^{\prime}\right)_{\mathrm{on}}^{-}-p_{\text {lon }}^{\prime-}-\left(k_{1}^{\prime}-p_{1}^{\prime}\right)_{\mathrm{on}}^{-}+i \frac{\varepsilon}{k_{1}^{\prime \prime}-p_{1}^{\prime+}}+i \frac{\varepsilon}{K_{f}^{++}-k_{1}^{\prime+}}+i \frac{\varepsilon}{p_{1}^{\prime+}}\right]} \text {. }
\end{aligned}
$$

In particular, the term generated by the first contribution in the square brackets can integrated over $k_{1}^{-}$taking the residue at the pole $k_{1 A}^{-}$. Then one gets

$$
\begin{aligned}
\left\langle k_{1}^{\prime+} \vec{k}_{1 \perp}^{\prime}\left|j^{c(1) \mu}\right| k_{1}^{+} \vec{k}_{1 \perp}\right\rangle_{(g)}= & i e_{1}\left(2 m_{1}\right)(i g)^{2} \Theta \frac{\theta\left(k_{1}^{\prime+}-p_{1}^{\prime+}\right)}{\left(k_{1}^{\prime+}-p_{1}^{\prime+}\right) p_{1}^{\prime+} \Delta_{f}^{(1 g)-} \Delta_{i}^{(1 g)-}} \Lambda_{+}\left(k_{1 \mathrm{on}}^{\prime}\right) \Gamma_{1}^{\alpha} \Lambda_{+}\left(p_{1 \mathrm{on}}^{\prime}\right) \gamma_{1}^{\mu} \Lambda_{+}\left(k_{1 \mathrm{on}}\right) \\
& \times \Lambda_{+}\left(\left(K_{f}-k_{1}^{\prime}\right)_{\mathrm{on}}\right) \Gamma_{2 \alpha} \Lambda_{+}\left(\left(K_{i}-k_{1}\right)_{\mathrm{on}}\right),
\end{aligned}
$$

where the combinations $\Delta_{f}^{(1 g)}$ and $\Delta_{i}^{(1 g)}$ of four momenta are

$$
\Delta_{f}^{(1 g)}=K_{f}-p_{1 \mathrm{on}}^{\prime}-\left(K_{f}-k_{1}^{\prime}\right)_{\mathrm{on}}-\left(k_{1}^{\prime}-p_{1}^{\prime}\right)_{\mathrm{on}}+i \varepsilon \quad \Delta_{i}^{(1 g)}=K_{i}-k_{\mathrm{on}}-\left(K_{f}-k_{1}^{\prime}\right)_{\mathrm{on}}-\left(k_{1}^{\prime}-p_{1}^{\prime}\right)_{\mathrm{on}}+i \varepsilon=\Delta_{i}^{(1 f)},
$$

since $k_{1}^{\prime}-p_{1}^{\prime}=p_{1}-k_{1}$. The second term in the square brackets yields a reducible contribution, which is canceled out by a term from $w^{(1)} g_{0} j^{c(0) \mu}$.

The result for the instantaneous diagram (h) can be obtained by taking the residue at the pole $k_{1 A}^{-}$in Eq. (F19), i.e.,

$$
\begin{aligned}
\left\langle k_{1}^{\prime+} \vec{k}_{1 \perp}^{\prime}\left|j^{c(1) \mu}\right| k_{1}^{+} \vec{k}_{1 \perp}\right\rangle_{(h)}= & \frac{i}{2} e_{1}(i g)^{2} \Theta \frac{\theta\left(k_{1}^{\prime+}-p_{1}^{\prime+}\right)}{\left(k_{1}^{\prime+}-p_{1}^{\prime+}\right) p_{1}^{\prime+} \Delta_{i}^{(1 g)-}} \Lambda_{+}\left(k_{1 \mathrm{on}}^{\prime}\right) \Gamma_{1}^{\alpha} \gamma_{1}^{+} \gamma_{1}^{\mu} \Lambda_{+}\left(k_{1 \mathrm{on}}\right) \Lambda_{+}\left(\left(K_{f}-k_{1}^{\prime}\right)_{\mathrm{on}}\right) \\
& \times \Gamma_{2 \alpha} \Lambda_{+}\left(\left(K_{i}-k_{1}\right)_{\mathrm{on}}\right) .
\end{aligned}
$$

\section{b. Diagram (i) for $p_{1}^{\prime+}>k_{1}^{\prime+}>0$}

In this kinematical region only an instantaneous contribution is an irreducible term, the one illustrated by diagram (i) in Fig. 3. By taking first the residue at the pole $k_{1 A}^{\prime-}$ and then the residue at the pole $k_{1 B}^{-}$, one has

$$
\begin{aligned}
\left\langle k_{1}^{\prime+} \vec{k}_{1 \perp}^{\prime}\left|j^{c(1) \mu}\right| k_{1}^{+} \vec{k}_{1 \perp}\right\rangle_{(i)}= & \frac{i}{2} e_{1}(i g)^{2} \Theta \frac{\theta\left(p_{1}^{\prime+}-k_{1}^{\prime+}\right)}{\left(p_{1}^{\prime+}-k_{1}^{\prime+}\right) p_{1}^{\prime+} \Delta_{f}^{(1 i)-}} \Lambda_{+}\left(k_{1 \mathrm{on}}^{\prime}\right) \Gamma_{1}^{\alpha} \gamma_{1}^{+} \gamma_{1}^{\mu} \Lambda_{+}\left(k_{\mathrm{lon}}\right) \Lambda_{+}\left(\left(K_{f}-k_{1}^{\prime}\right)_{\mathrm{on}}\right) \\
& \times \Gamma_{2 \alpha} \Lambda_{+}\left(\left(K_{i}-k_{1}\right)_{\mathrm{on}}\right)
\end{aligned}
$$


where the three-body denominator is the minus component of

$$
\Delta_{f}^{(1 i)}=K_{f}-k_{1 \mathrm{on}}^{\prime}-\left(K_{i}-k_{1}\right)_{\mathrm{on}}-\left(p_{1}^{\prime}-k_{1}^{\prime}\right)_{\mathrm{on}}=\Delta_{f}^{(1 a)},
$$

\section{APPENDIX G: $\mathcal{O}(1)$ CURRENT CONSERVATION IN THE LADDER YUKAWA MODEL}

The operatorial form of the current conservation at the first order of the QP expansion, as given by the expression in Eq. (74), will be explicitly checked in what follows. The symbol $\Theta=\theta\left(k_{1}^{+}\right) \theta\left(k_{1}^{\prime+}\right) \theta\left(K_{i}^{+}-k_{1}^{+}\right) \theta\left(K_{f}^{+}-k_{1}^{\prime+}\right)$ simplifies the notation. Furthermore, it is worth noting that the $\Delta$ 's, given in Eqs. (F8), (F12), (F14), (F22), and (F25), allow to express the momentum transfer, $Q$, in a convenient way, depending upon the term we are considering, and they have only minus components non vanishing.

Let us separate two kinematical regions: (i) $k_{1}^{\prime+}>Q^{+}$ and (ii) $Q^{+}>k_{1}^{\prime+}$.

$$
\text { 1. } p_{1}^{+}=k_{1}^{\prime+}-Q^{+}>0
$$

In this region one has contribution from diagrams (a), $\left(\mathrm{c}^{I}\right),(\mathrm{f}),(\mathrm{g}),(\mathrm{h})$ in Figs. 2 and 3 (since $k^{\prime+}-p_{1}^{\prime+}=p_{1}^{+}-$ $k_{1}^{+}>0$ ). For diagrams (a) and (g), the momentum transfer can be expressed in two different ways, viz.,

$$
\begin{aligned}
& Q=\Delta_{f}^{(1 a)}-\Delta_{i}^{(1 a)}+k_{1 \mathrm{on}}^{\prime}-p_{1 \mathrm{on}} \\
& Q=\Delta_{f}^{(1 g)}-\Delta_{i}^{(1 g)}-k_{\mathrm{lon}}+p_{\mathrm{lon}}^{\prime} .
\end{aligned}
$$

Therefore, since $\Lambda_{+}\left(k_{1 \text { on }}^{\prime}\right)\left(k_{1 \text { on }}^{\prime \prime}-\not p_{1 \text { on }}\right) \Lambda_{+}\left(p_{1 \text { on }}\right)=0$, one has

$$
\begin{aligned}
\Lambda_{+}\left(k_{1 \mathrm{on}}^{\prime}\right) \emptyset \Lambda_{+}\left(p_{1 \mathrm{on}}\right)= & \Lambda_{+}\left(k_{1 \mathrm{on}}^{\prime}\right) \frac{\gamma_{1}^{+}}{2} \Lambda_{+}\left(p_{1 \mathrm{on}}\right) \\
& \times\left(\Delta_{f}^{(1 a)-}-\Delta_{i}^{(1 a)-}\right) .
\end{aligned}
$$

Analogously one finds that,

$$
\begin{aligned}
\Lambda_{+}\left(p_{1 \mathrm{on}}^{\prime}\right) \emptyset \Lambda_{+}\left(k_{1 \mathrm{on}}\right)= & \Lambda_{+}\left(p_{1 \mathrm{on}}^{\prime}\right) \frac{\gamma_{1}^{+}}{2} \Lambda_{+}\left(k_{1 \mathrm{on}}\right) \\
& \times\left(\Delta_{f}^{(1 g)-}-\Delta_{i}^{(1 g)-}\right) .
\end{aligned}
$$

Equations (G3) and (G4) allow one to calculate the four divergence of the current.

The divergence for the term (a) plus (g) [see Eqs. (F7) and (F21)] is given by

$$
\begin{aligned}
Q \cdot\left\langle k_{1}^{\prime+} \vec{k}_{1 \perp}^{\prime}\left|j^{c(1)}\right| k_{1}^{+} \vec{k}_{1 \perp}\right\rangle_{(a)+(g)}= & \Theta i e_{1}(i g)^{2} \theta\left(k_{1}^{\prime+}-Q^{+}\right) \\
& \times\left[\frac{\theta\left(k_{1}^{+}-p_{1}^{+}\right)}{\left(k_{1}^{+}-p_{1}^{+}\right)}\left(\frac{1}{\Delta_{i}^{(1 a)-}}-\frac{1}{\Delta_{f}^{(1 a)-}}\right) \Lambda_{+}\left(k_{1 \mathrm{on}}^{\prime}\right) \frac{m_{1}}{p_{1}^{+}} \gamma_{1}^{+} \Lambda_{+}\left(p_{1 \mathrm{on}}\right) \Gamma_{1}^{\alpha} \Lambda_{+}\left(k_{\mathrm{lon}}\right)\right. \\
& \left.+\frac{\theta\left(k_{1}^{\prime+}-p_{1}^{\prime+}\right)}{\left(k_{1}^{\prime+}-p_{1}^{\prime+}\right)}\left(\frac{1}{\Delta_{i}^{(1 g)-}}-\frac{1}{\Delta_{f}^{(1 g)-}}\right) \Lambda_{+}\left(k_{1 \mathrm{on}}^{\prime}\right) \Gamma_{1}^{\alpha} \Lambda_{+}\left(p_{1 \mathrm{on}}^{\prime}\right) \frac{m_{1}}{p_{1}^{\prime+}} \gamma_{1}^{+} \Lambda_{+}\left(k_{1 \mathrm{on}}\right)\right] \\
& \times \Lambda_{+}\left(k_{2 \mathrm{on}}^{\prime}\right) \Gamma_{2 \alpha} \Lambda_{+}\left(k_{2 \mathrm{on}}\right),
\end{aligned}
$$

where we have used $k_{2 \text { on }}=\left(K_{i}-k_{1}\right)_{\text {on }}$ and $k_{2 \text { on }}^{\prime}=\left(K_{f}-k_{1}^{\prime}\right)_{\text {on }}$ to simplify the notation.

Let us consider the divergence of currents $\left(\mathrm{c}^{I}\right)$ and $\left(\mathrm{i}^{I}\right)$, corresponding to the contribution of instantaneous terms in the kinematical region $k_{1}^{\prime+}>Q^{+}>0$. The following relation, obtained by exploiting (i) the property $\left(\gamma^{+}\right)^{2}=0$ and (ii) the anticommutation rule $\left\{k, \gamma^{+}\right\}=2 k^{+}$, is useful for the next formal steps, i.e.,

$$
\begin{aligned}
\Lambda_{+}\left(k_{1 \mathrm{on}}^{\prime}\right) \emptyset \gamma^{+} & =\Lambda_{+}\left(k_{1 \mathrm{on}}^{\prime}\right)\left[k_{1}^{\prime}-\not p_{1}\right] \gamma^{+}=\Lambda_{+}\left(k_{1 \mathrm{on}}^{\prime}\right)\left[k_{1 \mathrm{on}}^{\prime}-\not p_{\text {1on }}\right] \gamma^{+}=\Lambda_{+}\left(k_{1 \mathrm{on}}^{\prime}\right)\left[m_{1}-\not p_{1 \mathrm{on}}\right] \gamma^{+} \\
& =\Lambda_{+}\left(k_{1 \mathrm{on}}^{\prime}\right)\left[-2 p_{1}^{+}+2 m_{1} \gamma^{+} \Lambda_{+}\left(p_{\text {1on }}\right)\right]=2 p_{1}^{+} \Lambda_{+}\left(k_{1 \mathrm{on}}^{\prime}\right)\left[-1+\frac{m_{1}}{p_{1}^{+}} \gamma^{+} \Lambda_{+}\left(p_{1 \mathrm{on}}\right)\right] .
\end{aligned}
$$

Then, from Eq. (F9), one has

$$
\begin{aligned}
Q \cdot\left\langle k_{1}^{\prime+} \vec{k}_{1 \perp}^{\prime}\left|j^{c(1)}\right| k_{1}^{+} \vec{k}_{1 \perp}\right\rangle_{(c)}^{I}= & \Theta i e_{1}(i g)^{2} \frac{\theta\left(k_{1}^{\prime+}-Q^{+}\right) \theta\left(k_{1}^{+}-p_{1}^{+}\right)}{\left(k_{1}^{+}-p_{1}^{+}\right) \Delta_{f}^{1 a-}} \Lambda_{+}\left(k_{1 \mathrm{on}}^{\prime}\right)\left[-1+\frac{m_{1}}{p_{1}^{+}} \gamma_{1}^{+} \Lambda_{+}\left(p_{1 \mathrm{on}}\right)\right] \\
& \times \Gamma_{1}^{\alpha} \Lambda_{+}\left(k_{1 \mathrm{on}}\right) \Lambda_{+}\left(k_{2 \mathrm{on}}^{\prime}\right) \Gamma_{2 \alpha} \Lambda_{+}\left(k_{2 \mathrm{on}}\right) .
\end{aligned}
$$

For diagram (i), Eq. (F24), in this region, a relation analogous to Eq. (G6) holds, i.e.,

$$
\gamma^{+} \emptyset \Lambda_{+}\left(k_{1 \mathrm{on}}\right)=\gamma^{+}\left[\not p_{1}^{\prime}-\not k_{1}\right] \Lambda_{+}\left(k_{1 \mathrm{on}}\right)=\gamma^{+}\left[\not p_{1 \mathrm{on}}^{\prime}-\not k_{1 \mathrm{on}}\right] \Lambda_{+}\left(k_{1 \mathrm{on}}\right)=2 p_{1}^{\prime+}\left[1-\frac{m_{1}}{p_{1}^{\prime+}} \Lambda_{+}\left(p_{1 \mathrm{on}}^{\prime}\right) \gamma^{+}\right] \Lambda_{+}\left(k_{1 \mathrm{on}}\right) .
$$

Therefore, from Eq. (F24), one obtains 


$$
\begin{aligned}
Q \cdot\left\langle k_{1}^{\prime+} \vec{k}_{1 \perp}^{\prime}\left|j^{c(1)}\right| k_{1}^{+} \vec{k}_{1 \perp}\right\rangle_{(i)}^{I}= & i \Theta e_{1}(i g)^{2} \frac{\theta\left(k_{1}^{\prime+}-Q^{+}\right) \theta\left(k_{1}^{+}-p_{1}^{+}\right)}{\left(k_{1}^{+}-p_{1}^{+}\right) \Delta_{f}^{(1 a)-}} \Lambda_{+}\left(k_{1 \mathrm{on}}^{\prime}\right) \Gamma_{1}^{\alpha}\left[1-\Lambda_{+}\left(p_{1 \mathrm{on}}^{\prime}\right) \frac{m_{1}}{p_{1}^{\prime+}} \gamma_{1}^{+}\right] \\
& \times \Lambda_{+}\left(k_{1 \mathrm{on}}\right) \Lambda_{+}\left(k_{2 \mathrm{on}}^{\prime}\right) \Gamma_{2 \alpha} \Lambda_{+}\left(k_{2 \mathrm{on}}\right) .
\end{aligned}
$$

Summing Eqs. (G7) and (G9), we find that

$$
\begin{aligned}
Q \cdot\left\langle k_{1}^{\prime+} \vec{k}_{1 \perp}^{\prime}\left|j^{c(1)}\right| k_{1}^{+} \vec{k}_{1 \perp}\right\rangle_{(c)+(i)}^{I}= & { } \Theta e_{1}(i g)^{2} \frac{\theta\left(k_{1}^{\prime+}-Q^{+}\right) \theta\left(k_{1}^{+}-p_{1}^{+}\right)}{\left(k_{1}^{+}-p_{1}^{+}\right) \Delta_{f}^{(1 a)-}} \Lambda_{+}\left(k_{1 \mathrm{on}}^{\prime}\right) \\
& \times\left[\frac{m_{1}}{p_{1}^{+}} \gamma_{1}^{+} \Lambda_{+}\left(p_{1 \mathrm{on}}\right) \Gamma_{1}^{\alpha}-\Gamma_{1}^{\alpha} \Lambda_{+}\left(p_{1 \mathrm{on}}^{\prime}\right) \frac{m_{1}}{p_{1}^{\prime+}} \gamma_{1}^{+}\right] \Lambda_{+}\left(k_{1 \mathrm{on}}\right) \Lambda_{+}\left(k_{2 \mathrm{on}}^{\prime}\right) \Gamma_{2 \alpha} \Lambda_{+}\left(k_{2 \mathrm{on}}\right) .
\end{aligned}
$$

The instantaneous terms (f) plus (h), see Eqs. (F11) and (F23) can be calculated following the same steps as the ones that lead to Eq. (G10). Noting that $\Delta_{i}^{(1 g)}=\Delta_{i}^{(1 f)}$, one has

$$
\begin{aligned}
Q \cdot\left\langle k_{1}^{\prime+} \vec{k}_{1 \perp}^{\prime}\left|j^{c(1)}\right| k_{1}^{+} \vec{k}_{1 \perp}\right\rangle_{(f)+(h)}= & \iota \Theta e_{1}(i g)^{2} \frac{\theta\left(k_{1}^{\prime+}-Q^{+}\right) \theta\left(p_{1}^{+}-k_{1}^{+}\right)}{\left(p_{1}^{+}-k_{1}^{+}\right) \Delta_{i}^{(1 g)-}} \Lambda_{+}\left(k_{1 \mathrm{on}}^{\prime}\right) \\
& \times\left[\frac{m_{1}}{p_{1}^{+}} \gamma_{1}^{+} \Lambda_{+}\left(p_{1 \mathrm{on}}\right) \Gamma_{1}^{\alpha}-\Gamma_{1}^{\alpha} \Lambda_{+}\left(p_{1 \mathrm{on}}^{\prime}\right) \frac{m_{1}}{p_{1}^{\prime+}} \gamma^{+}\right] \Lambda_{+}\left(k_{1 \mathrm{on}}\right) \Lambda_{+}\left(k_{2 \mathrm{on}}^{\prime}\right) \Gamma_{2 \alpha} \Lambda_{+}\left(k_{2 \mathrm{on}}\right) .
\end{aligned}
$$

Adding Eqs. (G5), (G10), and (G11) one gets in the kinematical region $k_{1}^{\prime+}>Q^{+}>0$ the result

$$
\begin{aligned}
Q \cdot\left\langle k_{1}^{\prime+} \vec{k}_{1 \perp}^{\prime}\left|j^{c(1)}\right| k_{1}^{+} \vec{k}_{1 \perp}\right\rangle \theta\left(k_{1}^{\prime+}-Q^{+}\right)= & \theta\left(k_{1}^{\prime+}-Q^{+}\right) \Theta\left[e_{1} \Lambda_{+}\left(k_{1 \mathrm{on}}^{\prime}\right) \frac{m_{1}}{p_{1}^{+}} \gamma_{1}^{+} \Lambda_{+}\left(p_{1 \mathrm{on}}\right)\left\langle p_{1}^{+} \vec{p}_{1 \perp}\left|w^{(1)}\left(K_{i}\right)\right| k_{1}^{+} \vec{k}_{1 \perp}\right\rangle\right. \\
& \left.-\left\langle k_{1}^{\prime+} \vec{k}_{1 \perp}^{\prime}\left|w^{(1)}\left(K_{f}\right)\right| p_{1}^{\prime+} \vec{p}_{1 \perp}^{\prime}\right\rangle e_{1} \Lambda_{+}\left(p_{1 \mathrm{on}}^{\prime}\right) \frac{m_{1}}{p_{1}^{\prime+}} \gamma_{1}^{+} \Lambda_{+}\left(k_{1 \mathrm{on}}\right)\right],
\end{aligned}
$$

where the matrix elements of the effective interaction in first order of QP truncation, for total momentum $K_{i}$, are given by

$$
\begin{aligned}
\left\langle p_{1}^{+} \vec{p}_{1 \perp}\left|w^{(1)}\left(K_{i}\right)\right| k_{1}^{+} \vec{k}_{1 \perp}\right\rangle= & i(i g)^{2} \theta\left(p_{1}^{+}\right) \theta\left(k_{1}^{+}\right) \theta\left(K_{i}^{+}-p_{1}^{+}\right) \theta\left(K_{i}^{+}-k_{1}^{+}\right)\left[\frac{\theta\left(p_{1}^{+}-k_{1}^{+}\right)}{\left(p_{1}^{+}-k_{1}^{+}\right)} \frac{1}{\Delta_{i}^{(1 g)-}}+\frac{\theta\left(k_{1}^{+}-p_{1}^{+}\right)}{\left(k_{1}^{+}-p_{1}^{+}\right)} \frac{1}{\Delta_{i}^{(1 a)-}}\right] \\
& \times \Lambda_{+}\left(p_{1 \mathrm{on}}\right) \Gamma_{1}^{\alpha} \Lambda_{+}\left(k_{1 \mathrm{on}}\right) \Lambda_{+}\left(k_{2 \mathrm{on}}^{\prime}\right) \Gamma_{2 \alpha} \Lambda_{+}\left(k_{2 \mathrm{on}}\right) .
\end{aligned}
$$

For total momentum $K_{f}$, the matrix elements of $w^{(1)}\left(K_{f}\right)$ can be easily obtained from the previous expression properly changing the individual momenta, i.e.,

$$
\begin{aligned}
\left\langle k_{1}^{\prime+} \vec{k}_{1 \perp}^{\prime}\left|w^{(1)}\left(K_{f}\right)\right| p_{1}^{\prime+} \vec{p}_{1 \perp}^{\prime}\right\rangle= & i(i g)^{2} \theta\left(p_{1}^{\prime+}\right) \theta\left(k_{1}^{\prime+}\right) \theta\left(K_{f}^{+}-p_{1}^{\prime+}\right) \theta\left(K_{f}^{+}-k_{1}^{\prime+}\right)\left[\frac{\theta\left(k_{1}^{\prime+}-p_{1}^{\prime+}\right)}{\left(k_{1}^{\prime+}-p_{1}^{\prime+}\right)} \frac{1}{\Delta_{f}^{(1 g)-}}+\frac{\theta\left(p_{1}^{\prime+}-k_{1}^{\prime+}\right)}{\left(p_{1}^{\prime+}-k_{1}^{\prime+}\right)} \frac{1}{\Delta_{f}^{(1 a)-}}\right] \\
& \times \Lambda_{+}\left(k_{1 \mathrm{on}}^{\prime}\right) \Gamma_{1}^{\alpha} \Lambda_{+}\left(p_{1 \mathrm{on}}^{\prime}\right) \Lambda_{+}\left(k_{2 \mathrm{on}}^{\prime}\right) \Gamma_{2 \alpha} \Lambda_{+}\left(k_{2 \mathrm{on}}\right) .
\end{aligned}
$$

Let us remind that $k_{1}^{\prime+}-p_{1}^{\prime+}=p_{1}^{+}-k_{1}^{+}$and that $\theta\left(p_{1}^{\prime+}\right)$ is redundant. For $\Gamma_{\alpha} \equiv 1$, i.e., for a scalar boson exchange, one obtains the expression derived in Ref. [10].

$$
\text { 2. } Q^{+}-\boldsymbol{k}_{1}^{\prime+}>\mathbf{0}
$$

In this kinematical region diagrams (b) and $\left(c^{I I}\right)$ contribute. Furthermore we consider the contribution of diagram (i) in this region [cf. the discussion after Eq. (F24)]. The divergence of the pair current [see, Eq. (F13)] is given by

$$
\begin{aligned}
Q \cdot\left\langle k_{1}^{\prime+} \vec{k}_{1 \perp}^{\prime}\left|j^{c(1)}\right| k_{1}^{+} \vec{k}_{1 \perp}\right\rangle_{(b)}= & -i \Theta e_{1}(i g)^{2} \frac{\theta\left(p_{1}^{\prime+}-k_{1}^{\prime+}\right)}{\left(p_{1}^{\prime+}-k_{1}^{\prime+}\right)} \frac{\theta\left(Q^{+}-k_{1}^{\prime+}\right)}{\Delta_{f}^{(1 a)-}} \Lambda_{+}\left(k_{\text {lon }}^{\prime}\right) \\
& \times \frac{m_{1}}{p_{1}^{+}} \gamma_{1}^{+} \Lambda_{+}\left(p_{1 \mathrm{on}}\right) \Gamma_{1}^{\alpha} \Lambda_{+}\left(k_{1 \mathrm{on}}\right) \Lambda_{+}\left(k_{2 \mathrm{on}}^{\prime}\right) \Gamma_{2 \alpha} \Lambda_{+}\left(k_{2 \mathrm{on}}\right),
\end{aligned}
$$

where $Q=\Delta_{\gamma}^{(1 b)}+k_{1 \text { on }}^{\prime}-p_{\text {1on }}$ has been used [see, Eq. (F14) and note that only $\Delta_{\gamma}^{(1 b)-}$ does not vanishes]. 
In the kinematical region under consideration, the divergence of the current (c) [see Eq. (F15)] is obtained by using Eq. (G6)

$$
\begin{aligned}
Q \cdot\left\langle k_{1}^{\prime+} \vec{k}_{1 \perp}^{\prime}\left|j^{c(1)}\right| k_{1}^{+} \vec{k}_{1 \perp}\right\rangle_{(c)}^{I I}= & i e_{1}(i g)^{2} \Theta \frac{\theta\left(p_{1}^{\prime+}-k_{1}^{\prime+}\right)}{\left(p_{1}^{\prime+}-k_{1}^{\prime+}\right)} \frac{\theta\left(Q^{+}-k_{1}^{\prime+}\right)}{\Delta_{f}^{(1 a)-}} \Lambda_{+}\left(k_{1 \mathrm{on}}^{\prime}\right)\left[\frac{m_{1}}{p_{1}^{+}} \gamma_{1}^{+} \Lambda_{+}\left(p_{1 \mathrm{on}}\right)-1\right] \\
& \times \Gamma_{1}^{\alpha} \Lambda_{+}\left(k_{1 \mathrm{on}}\right) \Lambda_{+}\left(k_{2 \mathrm{on}}^{\prime}\right) \Gamma_{2 \alpha} \Lambda_{+}\left(k_{2 \mathrm{on}}\right) .
\end{aligned}
$$

The divergence of the current (i), Eq. (F24), in this region, using Eq. (G8) results in

$$
\begin{aligned}
Q \cdot\left\langle k_{1}^{\prime+} \vec{k}_{1 \perp}^{\prime}\left|j^{c(1)}\right| k_{1}^{+} \vec{k}_{1 \perp}\right\rangle_{(i)}^{I I}= & i e_{1}(i g)^{2} \Theta \frac{\theta\left(p_{1}^{\prime+}-k_{1}^{\prime+}\right)}{\left(p_{1}^{\prime+}-k_{1}^{\prime+}\right)} \frac{\theta\left(Q^{+}-k_{1}^{\prime+}\right)}{\Delta_{f}^{(1 a)-}} \Lambda_{+}\left(k_{1 \mathrm{on}}^{\prime}\right) \Gamma_{1}^{\alpha}\left[1-\Lambda_{+}\left(p_{1 \mathrm{on}}^{\prime}\right) \frac{m_{1}}{p_{1}^{\prime+}} \gamma_{1}^{+}\right] \\
& \times \Lambda_{+}\left(k_{1 \mathrm{on}}\right) \Lambda_{+}\left(k_{2 \mathrm{on}}^{\prime}\right) \Gamma_{2 \alpha} \Lambda_{+}\left(k_{2 \mathrm{on}}\right) .
\end{aligned}
$$

where we have used that $Q=\Delta_{f}^{(1 g)}-\Delta^{(1 f)}+p_{\text {lon }}^{\prime}-k_{\text {lon }}$.

Finally, adding Eqs. (G15)-(G17), only the second term in the square bracket of (G17) survives, resulting in

$$
\begin{aligned}
& Q \cdot\left\langle k_{1}^{\prime+} \vec{k}_{1 \perp}^{\prime}\left|j^{c(1)}\right| k_{1}^{+} \vec{k}_{1 \perp}\right\rangle_{(b)+(c)+(i)} \theta\left(Q^{+}-k_{1}^{\prime+}\right) \\
& =-i e_{1}(i g)^{2} \Theta \frac{\theta\left(p_{1}^{\prime+}-k_{1}^{\prime+}\right)}{\left(p_{1}^{\prime+}-k_{1}^{\prime+}\right)} \frac{\theta\left(Q^{+}-k_{1}^{\prime+}\right)}{\Delta_{f}^{(1 a)-}} \Lambda_{+}\left(k_{1 \mathrm{on}}^{\prime}\right) \Gamma_{1}^{\alpha} \Lambda_{+}\left(p_{1 \mathrm{on}}^{\prime}\right) \Lambda_{+}\left(k_{2 \mathrm{on}}^{\prime}\right) \Gamma_{2 \alpha} \Lambda_{+}\left(k_{2 \mathrm{on}}\right) \Lambda_{+}\left(p_{1 \mathrm{on}}^{\prime}\right) \frac{m_{1}}{p_{1}^{\prime+}} \gamma_{1}^{+} \Lambda_{+}\left(k_{1 \mathrm{on}}\right) \\
& =\left\langle k_{1}^{\prime+} \vec{k}_{1 \perp}^{\prime}\left|w^{(1)}\left(K_{f}\right)\right| p_{1}^{\prime+} \vec{p}_{1 \perp}^{\prime}\right\rangle e_{1} \Lambda_{+}\left(p_{1 \mathrm{on}}^{\prime}\right) \frac{m_{1}}{p_{1}^{\prime+}} \gamma_{1}^{+} \Lambda_{+}\left(k_{1 \mathrm{on}}\right) \theta\left(Q^{+}-k_{1}^{\prime+}\right) .
\end{aligned}
$$

This single term corresponds to the interaction calculated at first order in QP expansion, for total momentum $K_{f}$, in the considered kinematical region. The term with $w^{(1)}\left(K_{i}\right)$ vanishes in this kinematical region, since we have $Q^{+}-k_{1}^{\prime+}=$ $-p_{1}^{+}>0$ and therefore we are outside the kinematical support of the interaction, see Eq. (G13).

The total divergence of the current operator is found by adding Eqs. (G12) and (G18) and $Q \cdot j^{c(o)}$. Then, by taking into account the definition of the left and right LF charge operator, Eqs. (64) and (66), one can obtain an expression for the LF WTI valid for the whole kinematical range $0<k_{1}^{\prime}<K_{f}^{+}$. This illustrates how one can check the formal expression for $Q \cdot j^{c(1)}$, given by Eq. (74), in the example of the Yukawa model in ladder approximation.

[1] F. Gross and D. O. Riska, Phys. Rev. C 36, 1928 (1987).

[2] S. Mandelstam, Proc. R. Soc. A 233, 248 (1955).

[3] F. Gross, Relativistic Quantum Mechanics and Field Theory (John Wiley \& Sons, New York 1993).

[4] T. Nieuwenhuis and J. A. Tjon, Phys. Rev. Lett. 77, 814 (1996).

[5] V. A. Karmanov and J. Carbonell, Eur. Phys. J. A 27, 11 (2006); Eur. Phys. J. A 27, 1 (2006).

[6] R. Alkofer, Braz. J. Phys. 37, 144 (2007).

[7] C. Itzykson and J.-B. Zuber, Quantum Field Theory (McGraw-Hill, International Edition, Singapore 1985).

[8] R. M. Woloshyn and A. D. Jackson, Nucl. Phys. B64, 269 (1973).

[9] J. H. O. Sales, T. Frederico, B. V. Carlson, and P. U. Sauer, Phys. Rev. C 61, 044003 (2000).

[10] J. H. O. Sales, T. Frederico, B. V. Carlson, and P. U. Sauer, Phys. Rev. C 63, 064003 (2001).

[11] T. Frederico, J. H. O. Sales, B. V. Carlson, and P. U. Sauer, Nucl. Phys. A737, 260c (2004).
[12] B. D. Keister and W. N. Polyzou, Adv. Nucl. Phys. 20, 225 (1991).

[13] J. Carbonell, B. Desplanques, V. A. Karmanov, and J.F. Mathiot, Phys. Rep. 300, 215 (1998).

[14] S. J. Brodsky, H.C. Pauli, and S. S. Pinsky, Phys. Rep. 301, 299 (1998).

[15] P. A. M. Dirac, Rev. Mod. Phys. 21, 392 (1949).

[16] J. A. O. Marinho, T. Frederico, and P. U. Sauer, Phys. Rev. D 76, 096001 (2007).

[17] D. S. Hwang and V. A. Karmanov, Nucl. Phys. B696, 413 (2004); V. A. Karmanov and D. S. Hwang, Few-Body Syst. 36, 155 (2005).

[18] A. N. Kvinikhidze and B. Blankleider, Phys. Rev. D 68, 025021 (2003).

[19] H.W. L. Naus, J.P.C. de Melo, and T. Frederico, FewBody Syst. 24, 99 (1998).

[20] F. M. Lev, E. Pace, and G. Salmè, Nucl. Phys. A641, 229 (1998).

[21] S. J. Brodsky and J. R. Primack, Ann. Phys. (N.Y.) 52, 315 (1969). 
[22] R. J. Perry, A. Harindranath, and K. G. Wilson, Phys. Rev. Lett. 65, 2959 (1990).

[23] S. Glazek, A. Harindranath, S. Pinsky, J. Shigemitsu, and K. Wilson, Phys. Rev. D 47, 1599 (1993).

[24] M. Mangin-Brinet, J. Carbonell, and V. A. Karmanov, Phys. Rev. D 64, 125005 (2001); Phys. Rev. C 68, 055203 (2003).

[25] J. R. Cooke, G. A. Miller, and D. R. Phillips, Phys. Rev. C 61, 064005 (2000).

[26] D. Lurié, A. J. MacFarlane and Y. Takahashi, Phys. Rev. 140, B1091 (1965).
[27] J. A. O. Marinho, T. Frederico, E. Pace, G. Salmè, and P. U. Sauer (to be published).

[28] C.-R. Ji, Y. Mishchenko, and A. Radyushkin, Phys. Rev. D 73, 114013 (2006).

[29] B. L. G. Bakker, J. K. Boomsma, and C.-R. Ji, Phys. Rev. D 75, 065010 (2007).

[30] P. G. Blunden, M. Burkardt, and G. A. Miller, Phys. Rev. C 59, R2998 (1999); Phys. Rev. C 60, 055211 (1999).

[31] G. A. Miller and R. Machleidt, Phys. Lett. B 455, 19 (1999); Phys. Rev. C 60, 035202 (1999). 